\title{
An Experimental Study of Seifert Fibered Dehn Surgery via SnapPea*
}

\author{
Kimihiko MOTEGI \\ Department of Mathematics, Nihon University, 3-25-40 Sakurajosui, Setagaya-ku, Tokyo 156-8550, Japan \\ E-mail: motegi@math.chs.nihon-u.ac.jp
}

Received July 1, 2002; final version accepted January 31, 2003

\begin{abstract}
Jeff Weeks' computer program SnapPea has been used widely in 3-manifold topology. This program computes hyperbolic structures after drillings and Dehn fillings on 3-manifolds, and it provides a variety of associated topological, geometric and arithmetic invariants.

In our previous study about Seifert fibered Dehn surgeries on knots, we used SnapPea to investigate a relationship between closed geodesics in hyperbolic knot complements and Seifert fibers after Seifert fibered surgeries on them. We will explain how we used SnapPea in the study and propose some questions inspired by the computer experiments. These experiments were carried out in the joint work with Katura Miyazaki while we were preparing the paper [Miyazaki and Motegi, Comm. Anal. Geom., 7: 551-582].
\end{abstract}

KEYWORDS: hyperbolic knot, Dehn surgery, Seifert fiber space, geodesic, computer program SnapPea

\section{Introduction}

Let $K$ be a knot in the 3-sphere $S^{3}$, with tubular neighborhood $N(K)$, and let $E(K)=S^{3}-$ int $N(K)$ be the exterior of $K$. Let $\gamma$ be a slope on $\partial E(K)$, i.e., the unoriented isotopy class of an essential simple loop on $\partial E(K)$. Let $(K ; \gamma)$ denote the closed orientable 3-manifold obtained from $S^{3}$ by $\gamma$-Dehn surgery on $K$, in other words, the result of attaching a solid torus $V$ to $E(K)$ so that $\gamma$ bounds a meridian disk of $V$ (Fig. 1.1). Dehn surgeries on knots can be naturally generalized to Dehn surgeries on links [25].

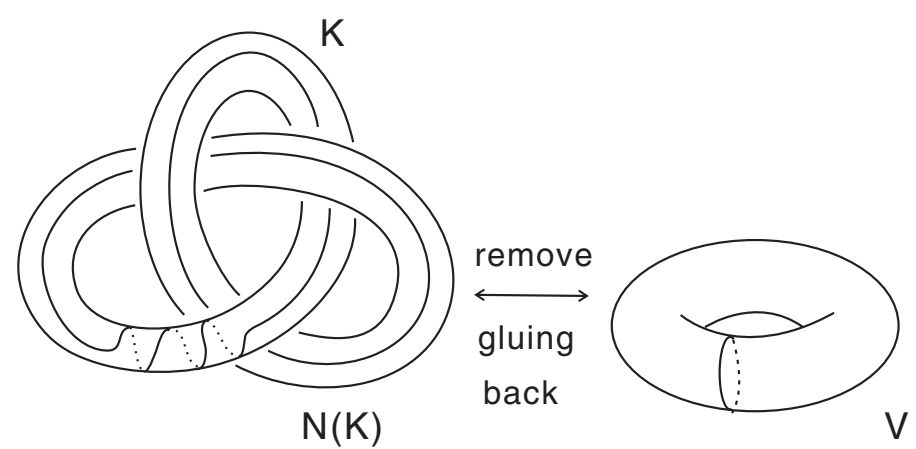

Fig. 1.1.

Using the preferred meridian-longitude pair of $K \subset S^{3}$, we parameterize slopes $\gamma$ of $K$ by $r=\frac{p}{q} \in \mathbb{Q} \cup\left\{\frac{1}{0}\right\}$; then we also write $(K ; r)$ for $(K ; \gamma)$. Note that $\left(K ; \frac{1}{0}\right) \cong S^{3}$ and $\frac{1}{0}$-surgery is called a trivial surgery.

Note that $H_{1}(E(K))$ is an infinite cyclic group generated by a meridian of $K$; a longitude of $K$ is homologous to zero in $E(K)$. Thus it is easy to see that $H_{1}\left(K ; \frac{p}{q}\right) \cong \mathbb{Z}_{|p|}$ for any knot $K$. In particular, $\frac{1}{n}$-surgery on $K$ yields a homology 3 sphere for any integer $n$.

By Thurston's uniformization theorem [24,28] and the torus theorem [15, 17], knots in the 3-sphere are divided into three classes: torus knots, satellite knots (i.e., a knot whose exterior contains a non-boundary-parallel, incompressible torus), and hyperbolic knots (i.e., a knot whose complement admits a complete hyperbolic structure of finite volume). Among these hyperbolic knots are the most important; and we empirically know that 'most' knots are hyperbolic.

Suppose that $K$ is a hyperbolic knot. Then the set of exceptional surgeries $\mathscr{E}_{K}=\{r \mid(K ; r)$ is not hyperbolic $\}$ is a finite set [27,28], and $\mathscr{E}_{K}$ can be expressed as $\mathscr{R}_{K} \cup \mathscr{T}_{K} \cup \mathscr{S}_{K} \cup \mathscr{C}_{K}$, where

$\mathscr{R}_{K}=\{r \mid(K ; r)$ is reducible, i.e., $(K ; r)$ contains a 2 -sphere not bounding a 3-ball $\}$,

$\mathscr{T}_{K}=\{r \mid(K ; r)$ is toroidal, i.e., $(K ; r)$ contains an incompressible torus $\}$,

* Supported in part by Grant-in-Aid for Scientific Research (No. 13640089), The Ministry of Education, Culture, Sports, Science and Technology, Japan.

2000 Mathematics Subject Classification: Primary 57M25, 57M50 Secondary 53C22, 57N10. 
$\mathscr{S}_{K}=\{r \mid(K ; r)$ is Seifert fibered $\}$, and

$\mathscr{C}_{K}=\{r \mid(K ; r)$ is a counter example to the geometrization conjecture $\}$.

By definition $\mathscr{S}_{K}$ contains a trivial surgery $\frac{1}{0}$. The geometrization conjecture [28] and the cabling conjecture [9] state that $\mathscr{C}_{K}=\emptyset$ and $\mathscr{R}_{K}=\emptyset$, respectively. Therefore it is expected that $\mathscr{E}_{K}=\mathscr{T}_{K} \cup \mathscr{S}_{K}$ for any hyperbolic knot $K$.

The simplest knot satisfying $\mathscr{T}_{K} \neq \varnothing$ and $\mathscr{S}_{K} \neq\left\{\frac{1}{0}\right\}$ is the figure-eight knot, for which we have $\mathscr{T}_{K}=\{0, \pm 4\}$ and $\mathscr{S}_{K}=\left\{\frac{1}{0}, \pm 1, \pm 2, \pm 3\right\}[27]$.

See [10] and [18] for surveys on Dehn surgery on knots.

In [22] we studied Seifert fibered surgeries from the viewpoint of "locating Seifert fibers" and we reported some experimental results obtained by using Weeks' computer program SnapPea [30]. The experiments suggested an interesting relationship between closed geodesics in hyperbolic knot complements and Seifert fibers after Seifert fibered surgeries on them. The main purpose in this paper is demonstrating how we got the experimental results via SnapPea.

It should be mentioned that there are many researches in which SnapPea plays important roles, say [14, 20].

SnapPea is available at http://humber.northnet.org/weeks/index/SnapPea.html.

\section{Acknowledgements}

I would like to take this opportunity to thank Jeff Weeks for providing the computer program SnapPea. Our study cannot be done without experiments using SnapPea. I would like to also thank Sadayoshi Kojima for introducing SnapPea while the work [22, Section 7] was being carried out. I would also like to thank the referees for careful reading and useful suggestions.

\section{How to Check Whether or Not a Given Knot or a Manifold Obtained by Dehn Surgery is Hyperbolic}

We begin by demonstrating how to check whether or not a given knot is hyperbolic.

\section{SnapPea Experiment 1 (Is a given knot hyperbolic?)}

Let us check that the figure-eight knot $K$ is hyperbolic.

(1) Choose New Link Projection in File menu (Picture 1.1). Then we have a window as in Picture 1.2.

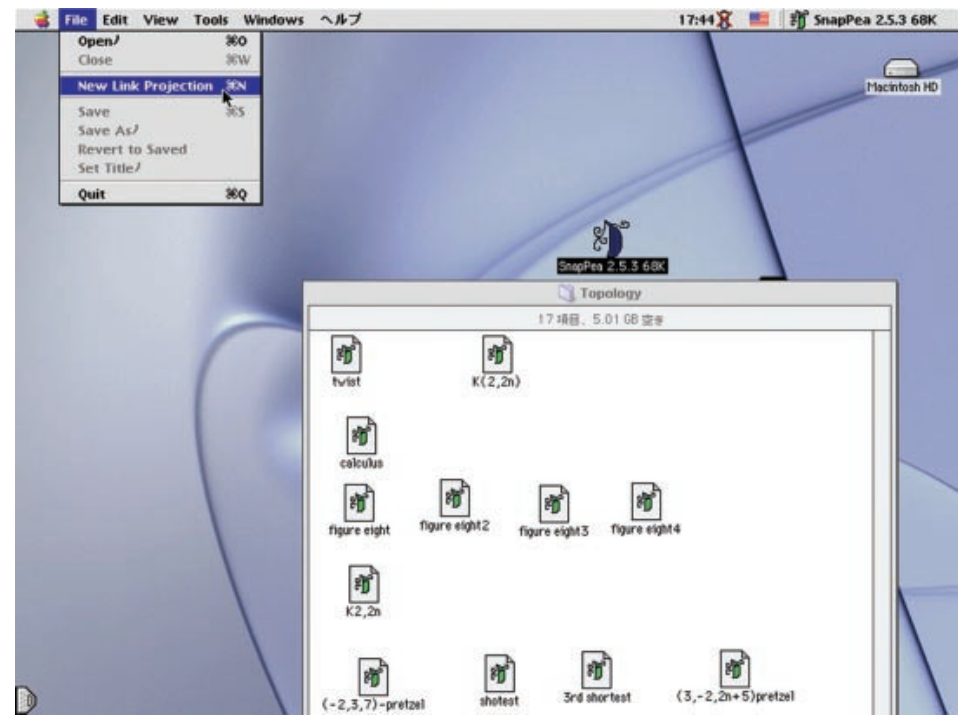

Picture 1.1.

(2) Draw a diagram of $K$ using a mouse; at the beginning, we do not need to care about an over/under crossing information (Picture 1.3).

To obtain the correct diagram of $K$, we invert some crossings by clicking on the crossing points as in Pictures 1.4 (before) and 1.5 (after).

(3) After correcting the diagram, click Complement button. Then we have a window as in Picture 1.6, which we call the "Dehn filling" window. In the window, SnapPea displays that $K$ is a hyperbolic knot with hyperbolic volume $2.029 \ldots$ and $H_{1}(E(K)) \cong H_{1}\left(S^{3}-K\right) \cong \mathbb{Z}$.

\section{SnapPea Experiment 2 (Is a Dehn surgered manifold hyperbolic?)}

We continue to investigate whether or not resulting manifolds obtained by Dehn surgeries on the figure-eight knot $K$ are hyperbolic. 


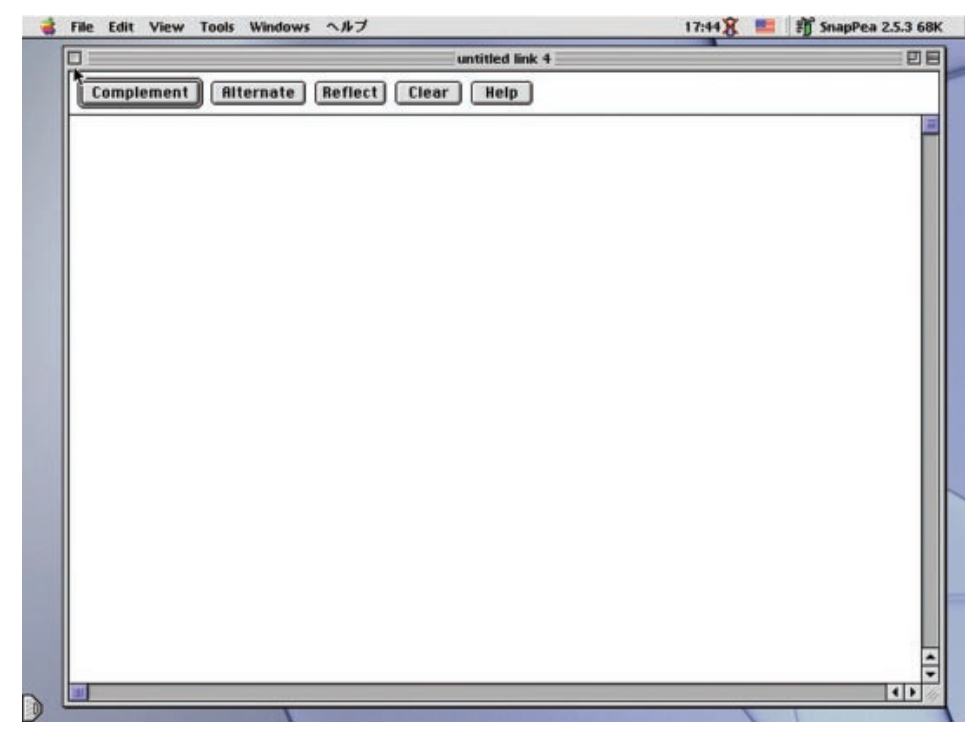

Picture 1.2.

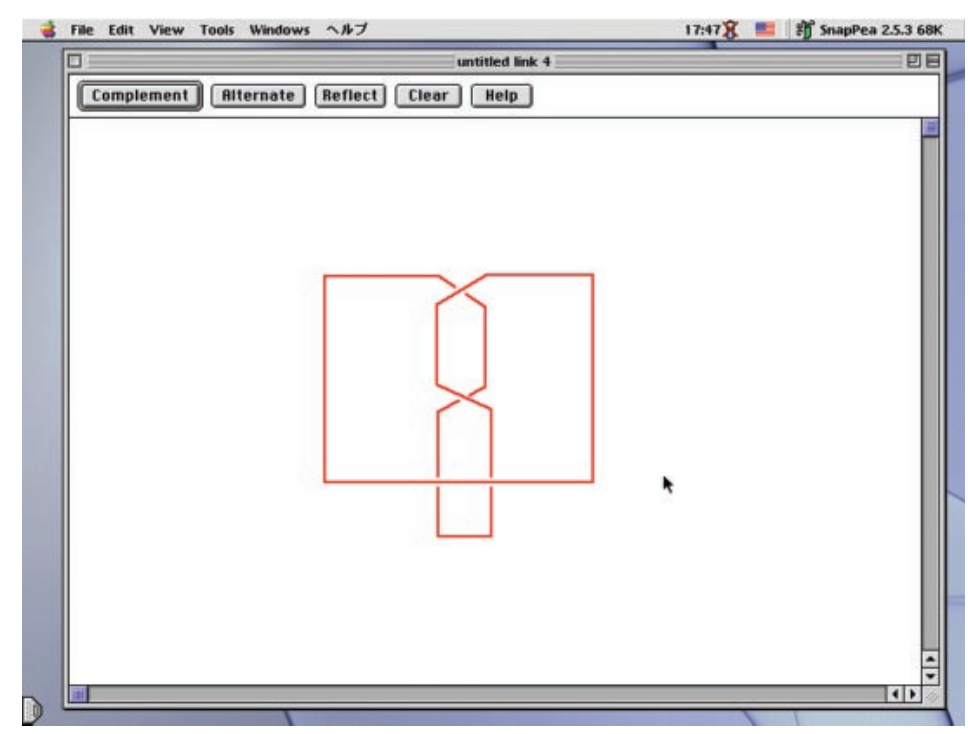

Picture 1.3.

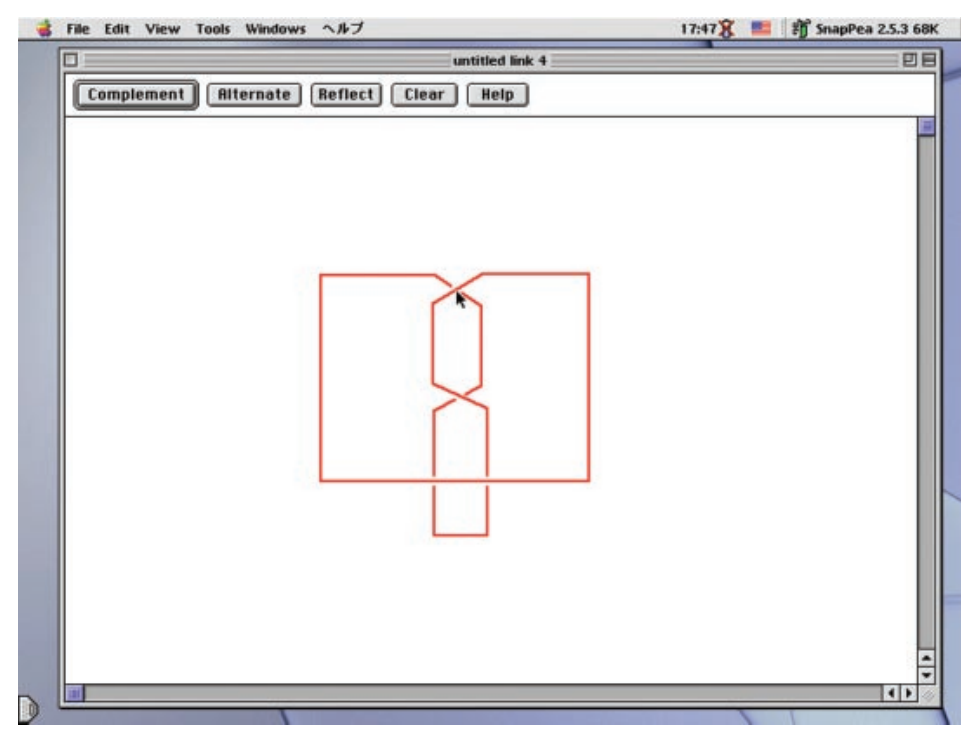

Picture 1.4. 


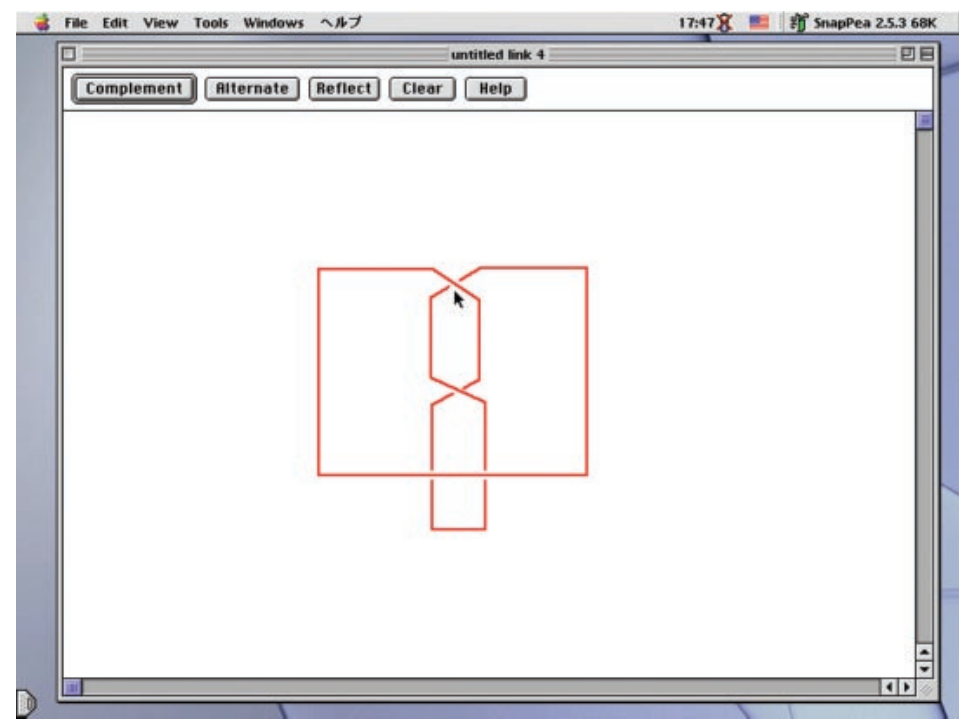

Picture 1.5.

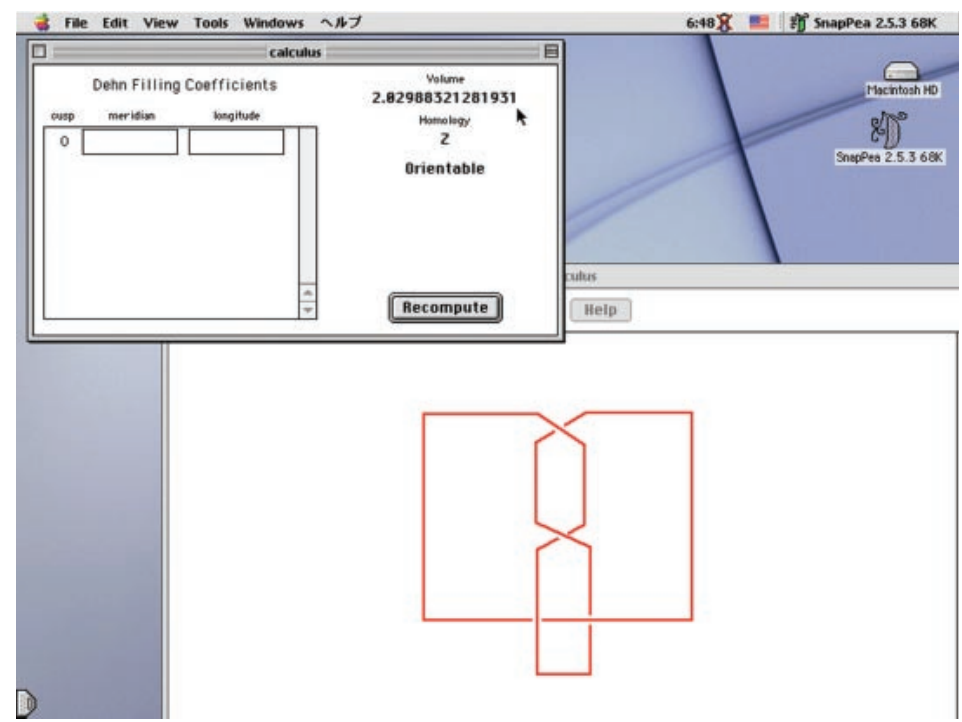

Picture 1.6.

(1) To perform $\frac{p}{q}$-surgery on $K$, we fill in the meridian box with $p$ and fill in the longitude box with $q$ in the "Dehn filling" window. For instance, in Picture 2.1 we fill in the meridian-longitude boxes with 1 and 5 respectively and then click Recompute button to get an information about the resulting manifold $\left(K ; \frac{1}{5}\right)$. Then SnapPea tells us that the result is also a hyperbolic manifold with hyperbolic volume $1.918 \ldots .$.

As we mentioned in Sect. $1, \mathscr{E}_{K}=\left\{\frac{1}{0}, 0, \pm 1, \pm 2, \pm 3, \pm 4\right\}$ for the figure-eight knot $K$.

What happens if the result is not hyperbolic?

(2) For instance, we perform -1-surgery on $K$, which can be done by filling in the meridian-longitude boxes with -1 and 1 respectively in the "Dehn filling" window as in Picture 2.2 and clicking Recompute button. Then as a result SnapPea displays a message suggesting that $(K ;-1)$ is a Seifert fiber space (or a solvable manifold) as in Picture 2.2.

In Proposition 4.3, we will show that $(K ;-1)$ is in fact a Seifert fiber space.

\section{Dehn Surgery on Knots with Surgery Descriptions}

In this section we will introduce a convenient method to investigate Dehn surgeries on (complicated) knots which contain some "twisted parts" as in Fig. 3.1.

Let us consider a link $K \cup c$ described in Fig. 3.2.

Since $-\frac{1}{n}$-surgery on the trivial component $c$ corresponds with $n$-twisting along the disk bounded by $c,-\frac{1}{n}$-surgery on $c$ yields $S^{3}$ and $K_{n}$ as the image of $K$. We say that $K_{n}$ is obtained from $K$ by $n$-twisting about $c$. So we can regard the 


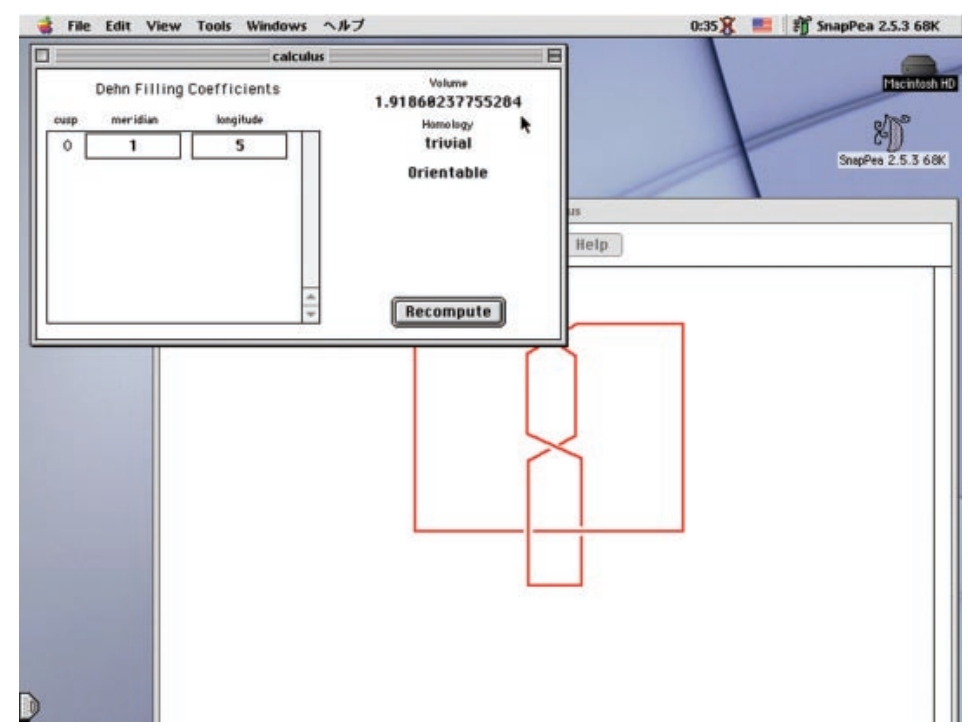

Picture 2.1.

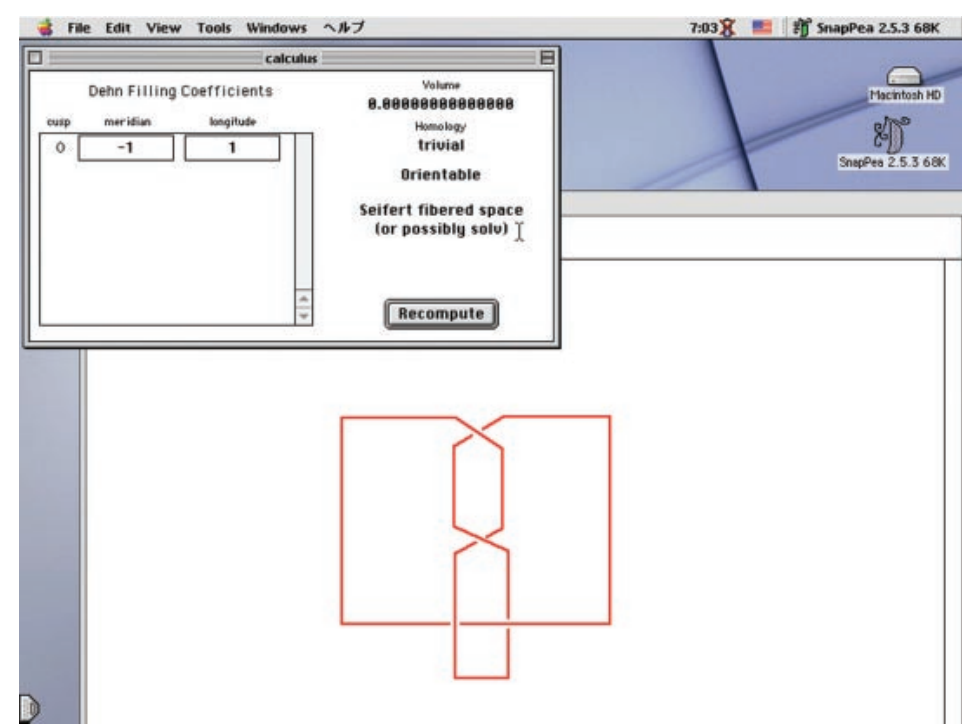

Picture 2.2.

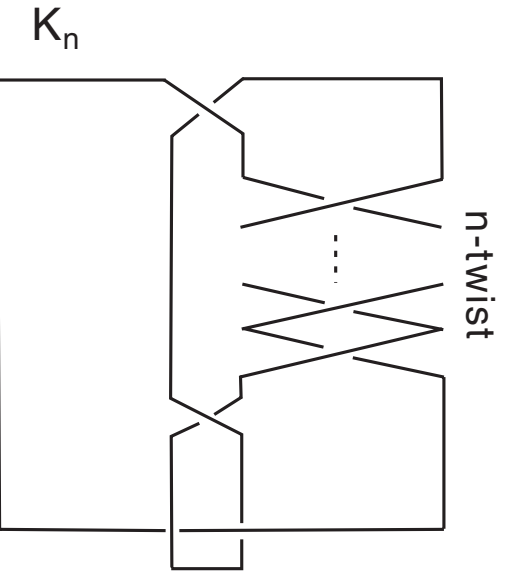

Fig. 3.1.

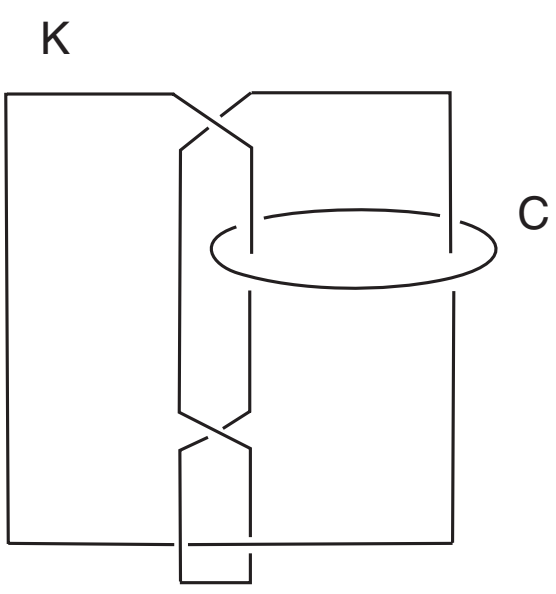

Fig. 3.2. 
link $K \cup c$ with $-\frac{1}{n}$-surgery on $c$ as $K_{n}$. More generally, by inserting trivial components $c_{1}, \ldots, c_{m}$, we can describe a knot with $m$ twisted parts. Such a description of the knot $K_{n}$ is called a surgery description of $K_{n}$.

\section{SnapPea Experiment 3 (Surgery descriptions of knots)}

Let $K \cup c$ be the link given by Fig. 3.2. In this experiment we consider a knot obtained from the figure-eight knot by twisting about $c$.

(1) We insert the trivial knot $c$ (the blue circle) as in Picture 3.1; first we do not care about an over/under crossing information and after closing the (blue) circle, by clicking on some crossing points, we convert the crossings to obtain the desired link $K \cup c$ (Picture 3.2).

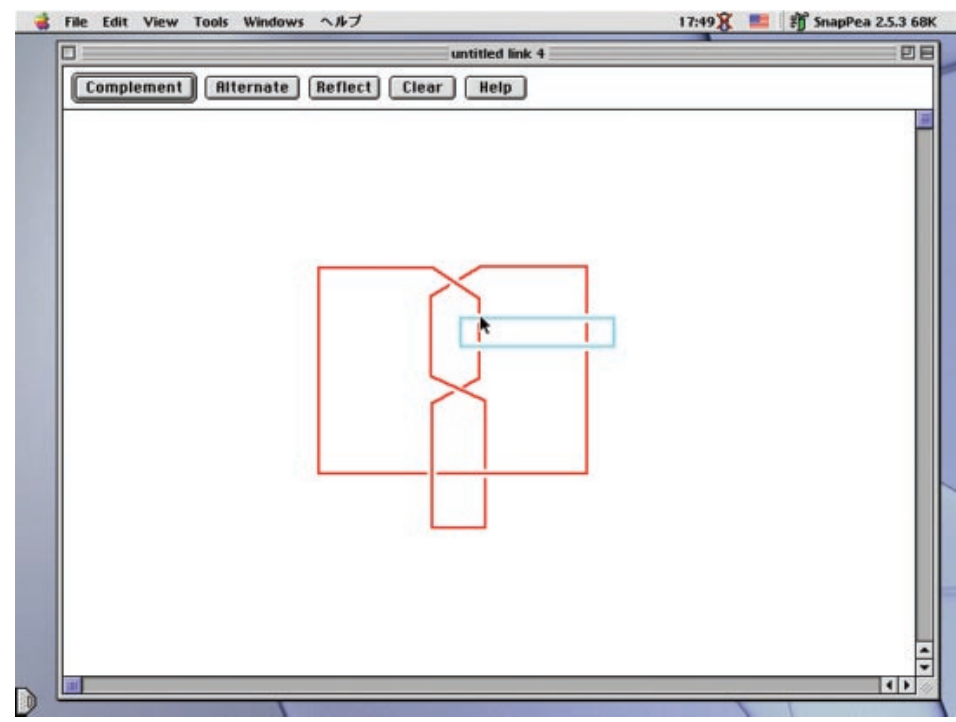

Picture 3.1.

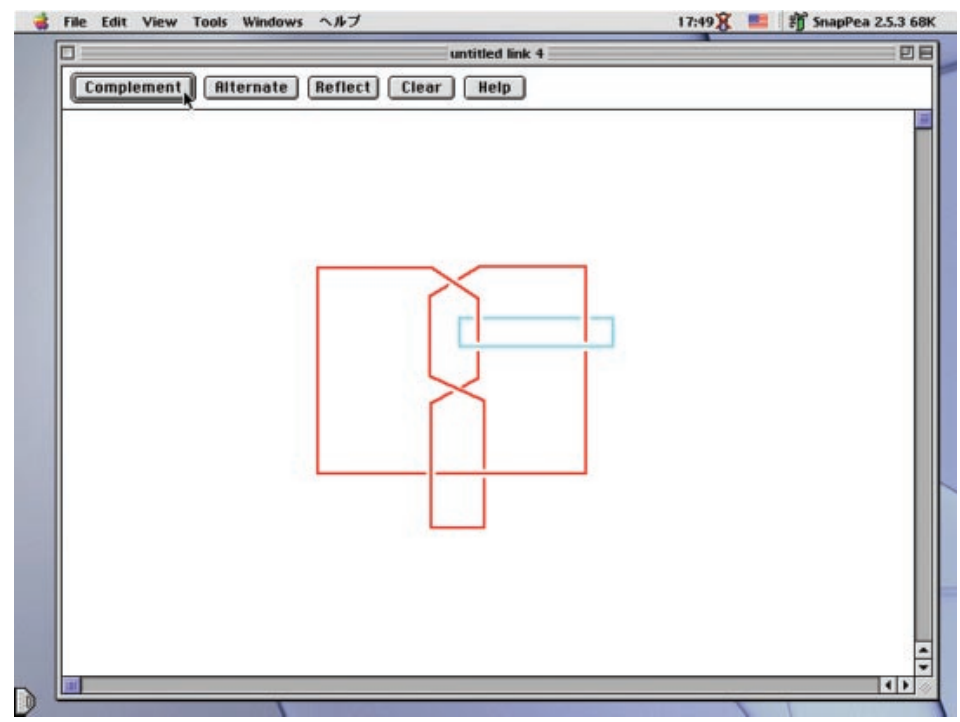

Picture 3.2.

(2) After drawing the diagram, click Complement button so that we have the "Dehn filling" window (Picture 3.3). Then SnapPea shows that the link $K \cup c$ is again hyperbolic.

(3) For instance, to obtain $K_{3}$, the knot obtained from $K$ by 3-twisting about $c$, we perform $-\frac{1}{3}$-surgery on $c$. This can be done by filling in the meridian-longitude boxes in the second row with 1 and -3 respectively as in Picture 3.4 and clicking Recompute button. Then SnapPea displays a message showing that the knot $K_{3}$ is also a hyperbolic knot with hyperbolic volume $5.114 \ldots$...

Let us consider Dehn surgeries on knots $K_{n}$ given by surgery descriptions. To perform $\frac{p}{q}$-surgery on $K_{n}$ correctly, we need to consider the effect of the twisting on the surgery coefficient. 


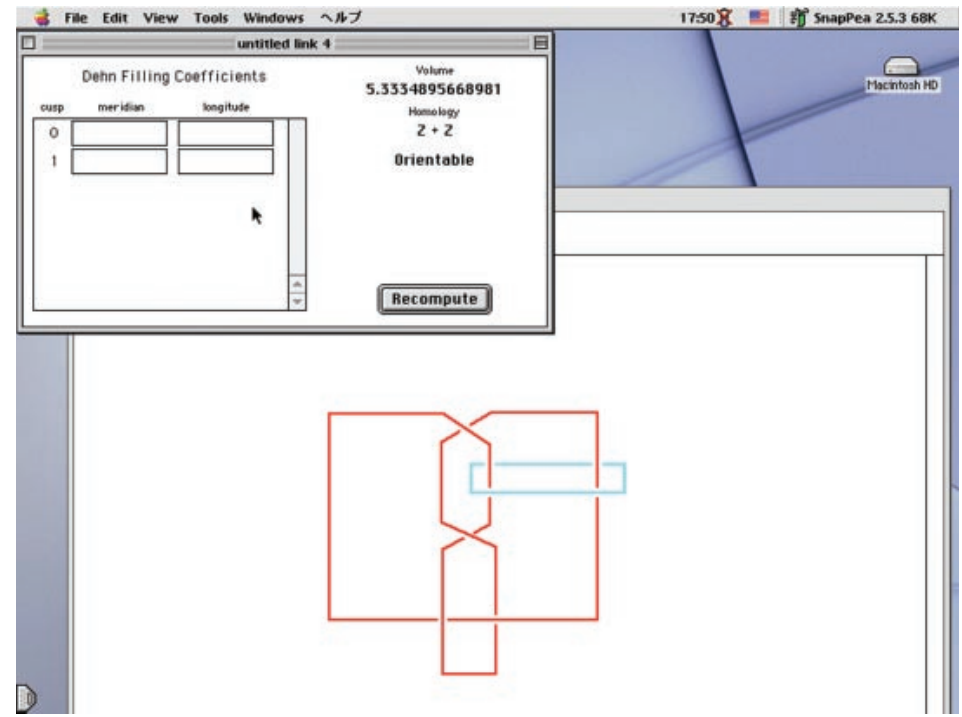

Picture 3.3.

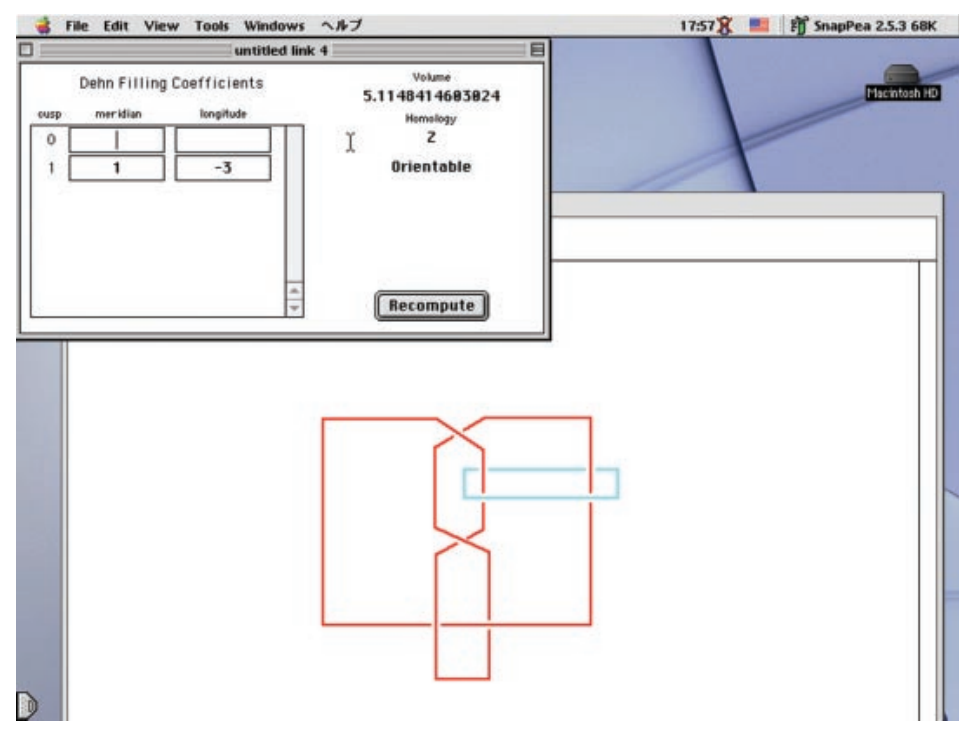

Picture 3.4.

Proposition 3.1 ([25]). Let $K \cup c$ be a link in $S^{3}$ such that $c$ is a trivial knot. Let $K_{n}$ be a knot obtained from $K$ by $n$ twisting about $c$. Then the manifold obtained by $\frac{p}{q}$-surgery on $K$ and $-\frac{1}{n}$-surgery on $c$ is homeomorphic to the result of $\frac{p}{q}+n[\ell k(K, c)]^{2}$-surgery on $K_{n}$, where $\ell k(K, c)$ denotes the linking number of $K$ and $c$.

\section{SnapPea Experiment 4 (Surgery on knots with surgery descriptions)}

We start with Picture 3.4; recall that $K$ is the red component and $c$ is the blue component.

(1) Compute the linking number of $K$ and $c$, see [25, Chapt. 5]. In our example $\ell k(K, c)=2$ with suitable orientations of $K$ and $c$; the choice of their orientations is irrelevant because we need the square of the linking number, see Proposition 3.1.

(2) For instance, we perform 2-surgery on $K_{3}$. Then the required surgery coefficient on $K$ is $\frac{p}{q}=2-3 \times 2^{2}=-10$ (Proposition 3.1). Thus we fill in the meridian-longitude boxes in the first row with -10 and 1 respectively and click Recompute button (Picture 4.1). Then SnapPea shows that the result $\left(K_{3} ; 2\right)$ is a hyperbolic manifold with hyperbolic volume $4.955 \ldots$ and $H_{1}\left(\left(K_{3} ; 2\right)\right) \cong \mathbb{Z}_{2}$ (Picture 4.1$)$.

In practice, when we study knots obtained from a simpler knot by twistings about a trivial knot and surgeries on them, the above method is quite useful.

Exercise. Check that $-\frac{23}{327}$-surgery on $K_{2002}$ produces a hyperbolic 3-manifold via SnapPea. 


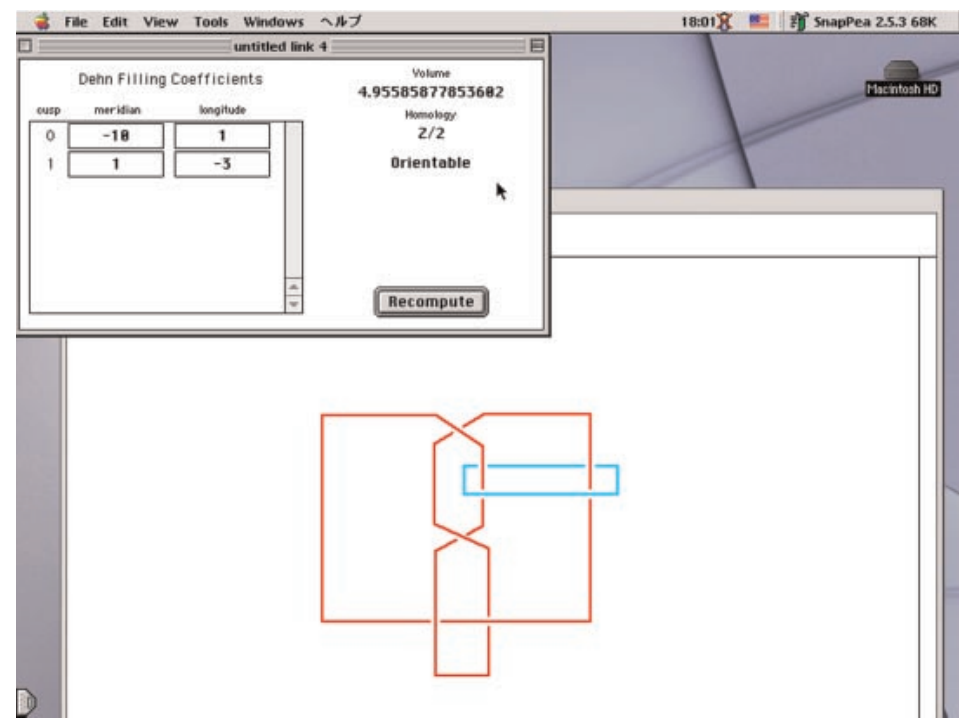

Picture 4.1.

\section{Which Curves in Knot Complements become Fibers after Seifert Fibered Surgeries?}

This section is based on the joint work with Katura Miyazaki [22] in which we studied Seifert fibered surgeries from the view point of "locating Seifert fibers".

Question 1. Suppose that $(K ; r)$ is a Seifert fiber space. If a fiber in $(K ; r)$ is contained in $S^{3}-\operatorname{int} N(K)$, then it can be regarded as a knot in $S^{3}$. Which knot $c$ in $S^{3}$, then, becomes a fiber in $(K ; r)$ ?

To get a feel we start with some examples.

Example (Seifert fibered surgeries on torus knots). Let $K$ be a torus knot on the boundary of an unknotted solid torus in $S^{3}$, and let $c$ be the core of the solid torus. If $(K ; r)$ is a Seifert fiber space, it admits a Seifert fibration in which the trivial knot $c$ is a fiber in $(K ; r)$.

Example (Seifert fibered surgeries on satellite knots). Let $K$ be a satellite knot such that $(K ; r)$ is Seifert fibered. If $\pi_{1}((K ; r))$ is finite or $(K ; r)$ is non-simple, then [2] or [21], respectively shows that a torus knot is a companion of $K$; the Seifert fibration of the torus knot exterior extends over $(K ; r)$. Thus, as in Example above a trivial knot in $S^{3}$ becomes a fiber in $(K ; r)$.

In these examples, there is a natural answer to Question 1. Thus we are interested in Question 1 for Seifert fibered surgeries on hyperbolic knots.

Example (Seifert fibered surgeries on the figure-eight knot). Let $K$ be the figure-eight knot. Then $(K ; r)$ is a Seifert fiber space if and only if $r=\frac{1}{0}, \pm 1, \pm 2, \pm 3$. In Table $1, S^{2}\left(m_{1}, m_{2}, m_{3}\right)$ denotes a Seifert fiber space over $S^{2}$ with three exceptional fibers of indices $m_{1}, m_{2}$ and $m_{3}$.

Table 1. Exceptional surgeries on the figure-eight knot

\begin{tabular}{|c|c|c|c|c|}
\hline & $r=\frac{1}{0}$ & $r= \pm 1$ & $r= \pm 2$ & $r= \pm 3$ \\
\hline$(K ; r)$ & $S^{3}$ & Seifert & Seifert & Seifert \\
& & $S^{2}(2,3,7)$ & $S^{2}(2,4,5)$ & $S^{2}(3,3,4)$ \\
\hline
\end{tabular}

We will show that the trivial knot $c_{1}$ in Fig. 4.1 becomes an exceptional fiber in some Seifert fibration of $(K ; r)$ for $r=-1,-2,-3$. The trivial knot $c_{2}$ also has this property.

\section{SnapPea Experiment 5 (Seifert fibered surgeries on the figure-eight knot I)}

(1) Following SnapPea Experiment 1 (1) (2), we draw the diagram of $K \cup c_{1}$ as in Picture 5.1.

(2) After drawing the diagram, click Complement button. Then fill in the meridian-longitude boxes in the second row with 1 and 0 respectively, and then click Recompute button to recover the figure knot complement 


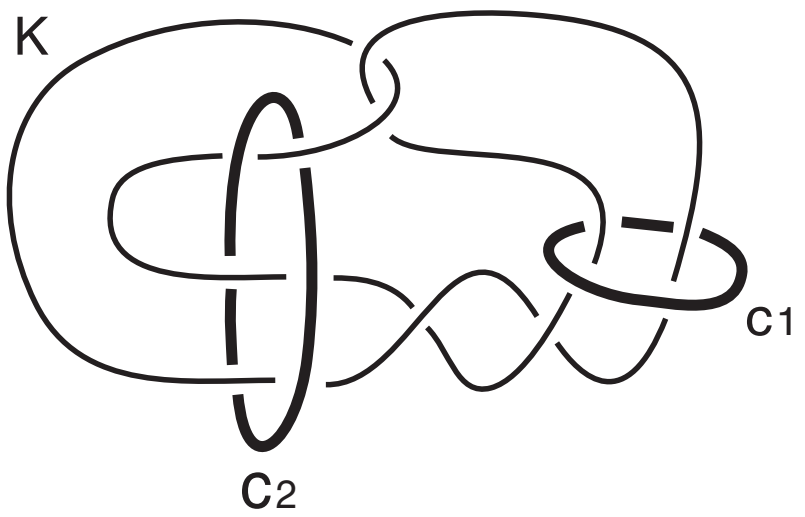

Fig. 4.1.

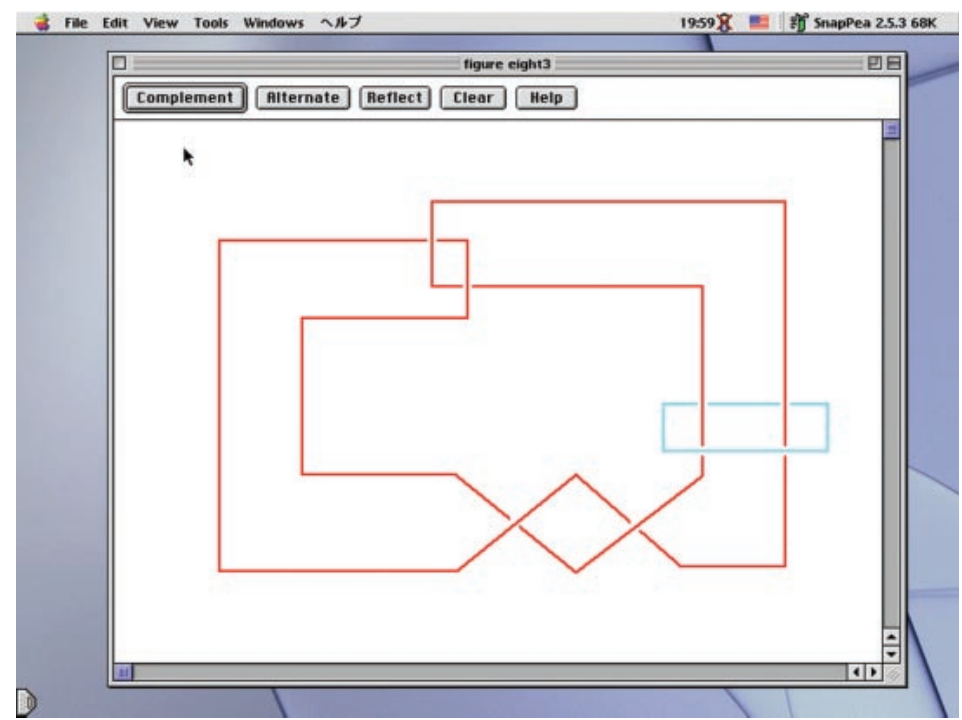

Picture 5.1.

(Picture 5.2). On the picture SnapPea shows that the figure eight knot is a hyperbolic knot with hyperbolic volume $2.029 \ldots$

(3) To perform -1-surgery on $K$, we fill in the meridian-longitude boxes in the first row with -1 and 1 respectively as in Picture 5.3 and click Recompute button. Then SnapPea suggests that the result is not hyperbolic, compare with Picture 2.2.

To recognize that $(K ;-1)$ is a Seifert fiber space, we need a proper proof. Later in Proposition 4.3 we will give a proper proof of the fact that $(K ;-1)$ is a Seifert fiber space over $S^{2}$ with three exceptional fibers of indices 2 , 3 and 7 , and hereafter we assume this fact.

(4) How to check that $c_{1}$ becomes a fiber in $(K ;-1)$ via SnapPea.

We need the following fact.

Lemma 4.1. Let $K$ be an arbitrary knot in $S^{3}$. Suppose that $(K ; r)\left(¥ S^{2} \times S^{1}\right)$ is a Seifert fiber space and $c$ is a knot in $(K ; r)$. If $(K ; r)-\operatorname{int} N(c)$ is Seifert fibered, then $(K ; r)$ admits a Seifert fibration so that $c$ is a fiber.

Proof of Lemma 4.1. Since $(K ; r) \neq S^{2} \times S^{1}$ (by the assumption) and $(K ; r) \neq \mathbb{R} P^{3} \sharp \mathbb{R} P^{3}$ for homological reason, by [16, Lemma VI.7], $(K ; r)$ is irreducible.

If the meridian of $N(c)$ is not a fiber in the Seifert fibration of $(K ; r)-\operatorname{int} N(c)$, then we can extend it to a Seifert fibration of $(K ; r)$ so that $c$ is a fiber as desired. So in the following we assume that the meridian is a fiber in the Seifert fibration of $(K ; r)-\operatorname{int} N(c)$.

If the base orbifold $B$ of $(K ; r)-\operatorname{int} N(c)$ is a disk with at most one cone point, then $(K ; r)-\operatorname{int} N(c)$ is a solid torus. Then by changing the Seifert fibration so that the meridian of $N(c)$ is not a fiber, we reduce to the above situation. Otherwise, we have a properly embedded arc in $B$ which does not cut off a disk without cone points. This implies that $(K ; r)$ is reducible, a contradiction. 


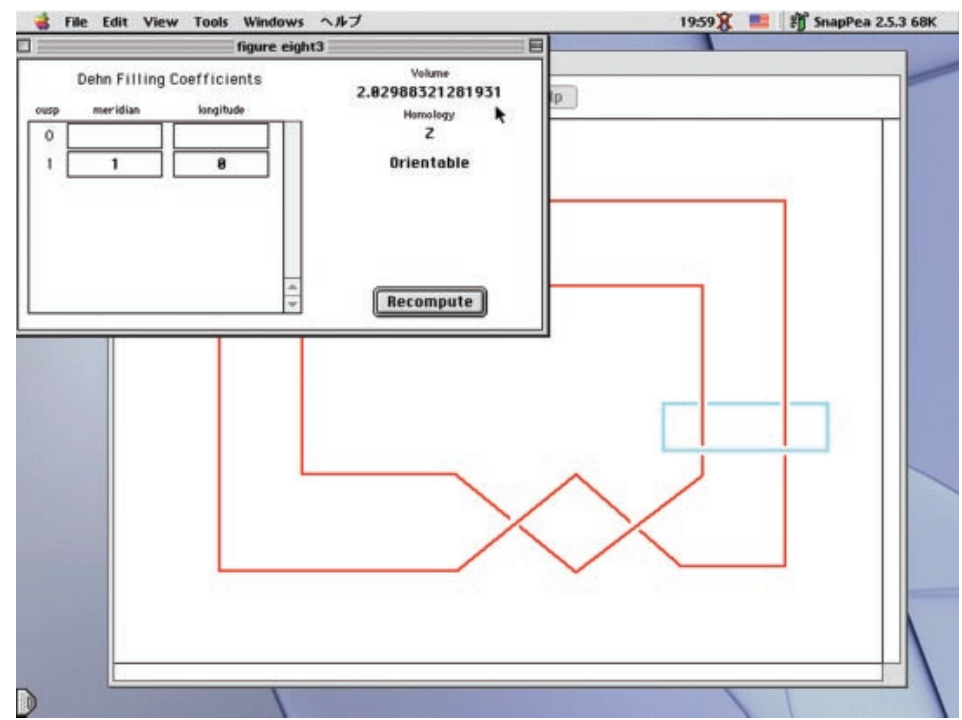

Picture 5.2.

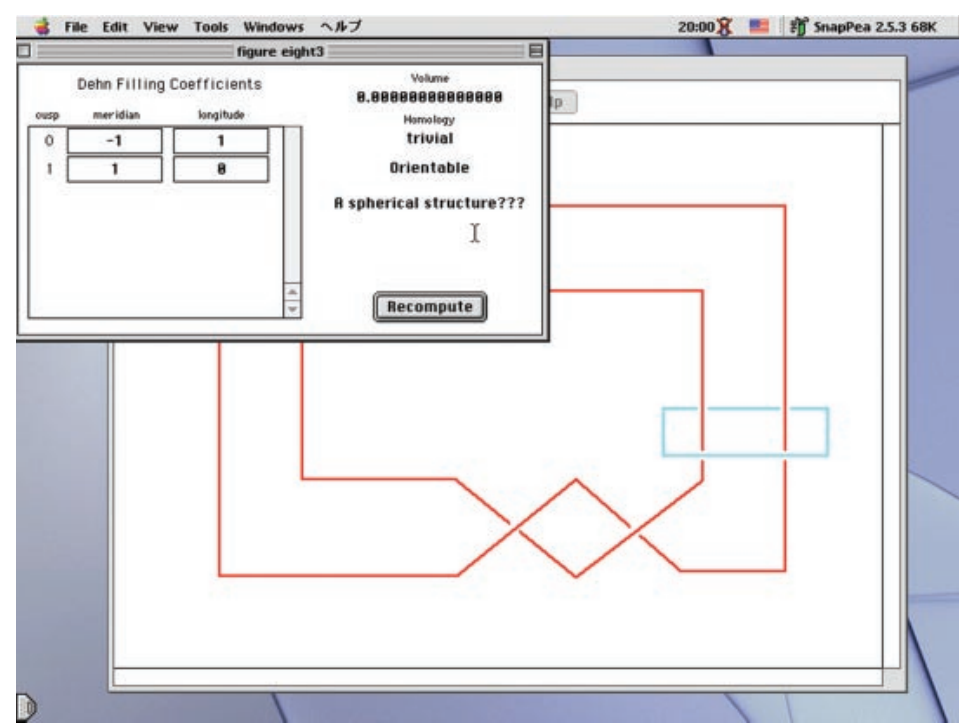

Picture 5.3.

In Lemma 4.1, if a Seifert fibration of $(K ; r)$ is unique up to isotopy, then we can isotope the Seifert fibration of $(K ; r)$ so that $c$ becomes a fiber.

(5) To drill out $c_{1}$ from $(K ;-1)$, we delete 1 and 0 from the second row as in Picture 5.4 and click Recompute button. Then SnapPea suggests that the result is Seifert fibered (or possibly solvable).

(6) Choose Fundamental Group in View menu as in Picture 5.5.

Then SnapPea computes the fundamental group $G=\pi_{1}\left((K ;-1)-c_{1}\right) \cong \pi_{1}\left((K ;-1)-\right.$ int $\left.N\left(c_{1}\right)\right)$ and gives us the following presentation (Picture 5.6):

$$
G=\left\langle a, b \mid a^{2} b^{3}\right\rangle .
$$

From the presentation we see that $a^{2}=b^{-3}$ belongs to the center of $G$. Furthermore, $a^{2}=b^{-3}$ generates an infinite cyclic normal subgroup of $G$. For otherwise, $\left(a^{2}\right)^{n}=\left(b^{-3}\right)^{n}=1$ for some integer $n>0$ and the abelianization of $G$ would be a finite group, a contradiction. Since $(K ;-1)$ is assumed to be Seifert fibered over $S^{2}$ with three exceptional fibers, it is irreducible [16, Example VI.13]. Thus if $(K ;-1)-\operatorname{int} N\left(c_{1}\right)$ is reducible, then there is a 3 -ball in $(K ;-1)$ containing $c_{1}$ in its interior, and hence $(K ;-1)-\operatorname{int} N\left(c_{1}\right) \cong(K ;-1) \sharp\left(S^{3}-\operatorname{int} N\left(c_{1}\right)\right)$. This implies that $G$ is a free product of two nontrivial groups. Since the center of the free product of any two nontrivial groups consists of the identity element alone, we should have $a^{2}=b^{-3}=1$ in $G$, a contradiction. It follows that $(K ;-1)-\operatorname{int} N\left(c_{1}\right)$ is an irreducible 3-manifold with infinite cyclic normal subgroup. Apply the following characterization theorem to conclude that $(K ;-1)-\operatorname{int} N\left(c_{1}\right)$ is Seifert fibered. 


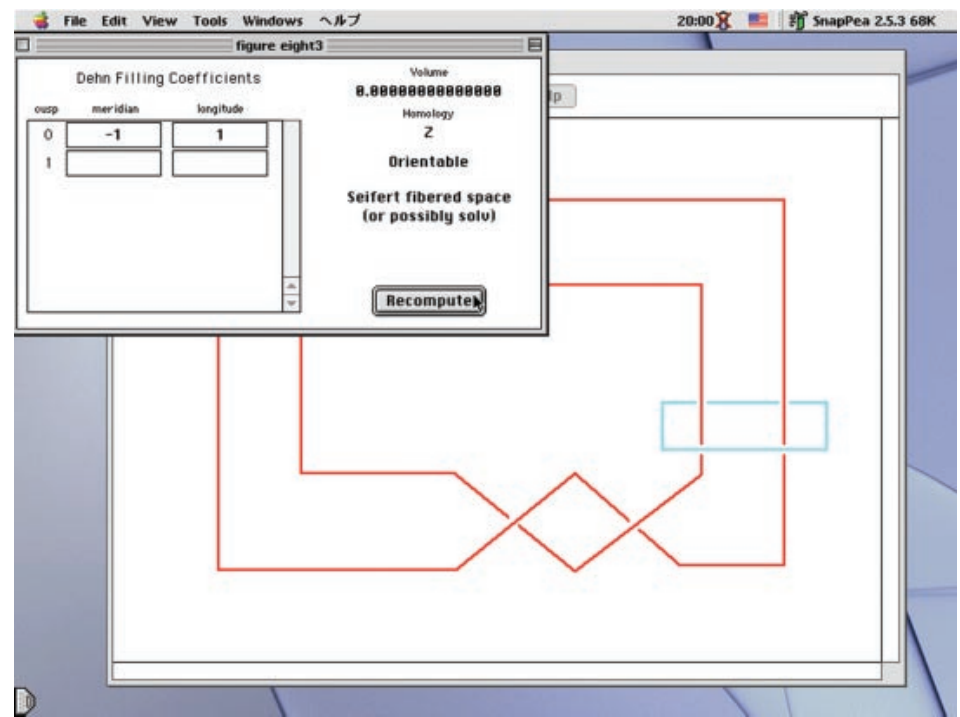

Picture 5.4.

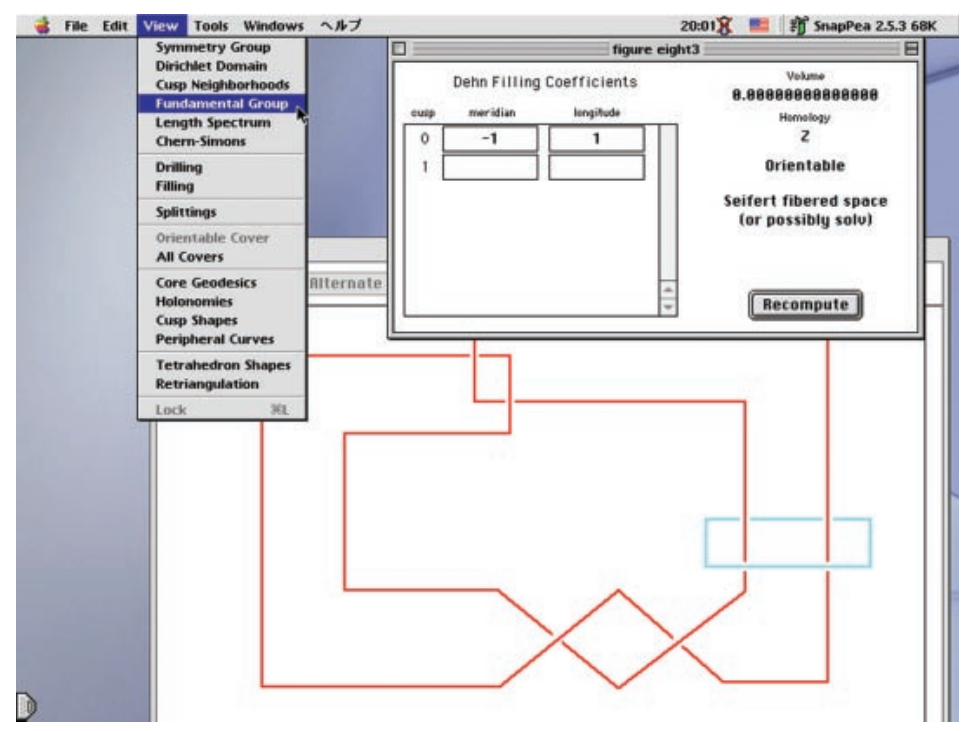

Picture 5.5.

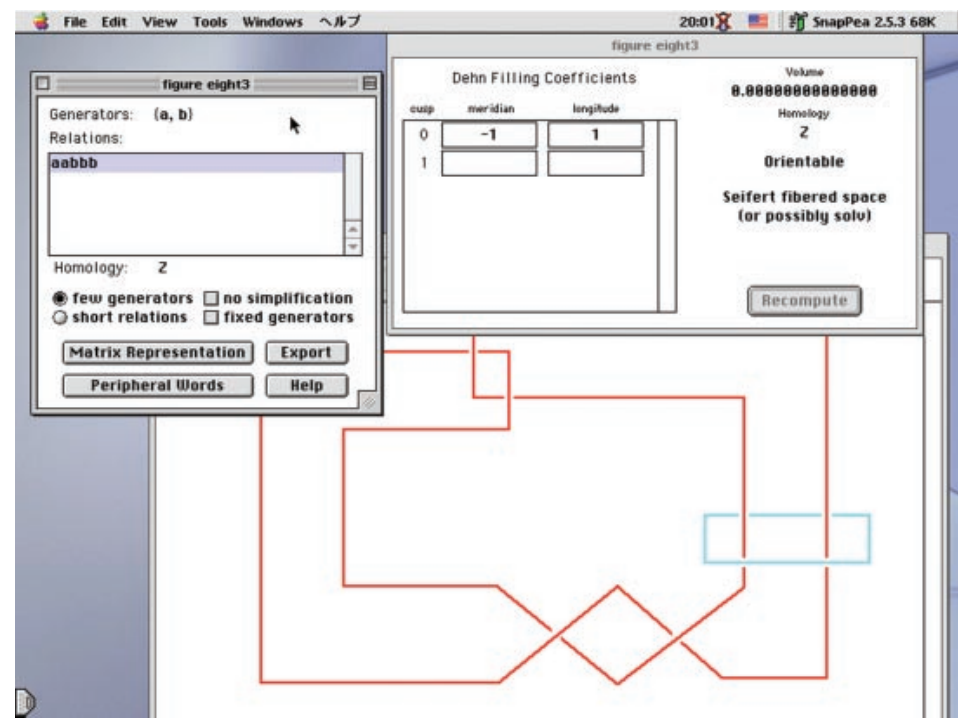

Picture 5.6. 
Theorem 4.2 (Seifert fiber space conjecture $[8,4])$. Let $M$ be an irreducible 3-manifold with infinite $\pi_{1}$. Then $M$ is Seifert fibered if and only if $\pi_{1}(M)$ has an infinite, cyclic, normal subgroup.

In our situation we need only a partial solution of the Seifert fiber space conjecture settled by Waldhausen [29], Gordon-Heil [11], see also Jaco-Shalen [15].

From Lemma 4.1, we see that $c_{1}$ is a fiber in some Seifert fibration of $(K ;-1)$.

Furthermore by assuming that $(K ;-1)$ is a Seifert fiber space over $S^{2}$ with three exceptional fibers of indices 2,3 and 7 , the presentation suggests that the exterior $(K ;-1)-\operatorname{int} N\left(c_{1}\right)$ is a Seifert fiber space over the disk with two exceptional fibers of indices 2, 3, and hence the index of $c_{1}$ is 7 . We will give a proper proof of these facts in Proposition 4.3.

Recall that $(K ;-2)($ resp. $(K ;-3))$ is a Seifert fiber space over $S^{2}$ with three exceptional fibers of indices 2,4 and 5 (resp. 3, 3 and 4).

(7) Applying the same method as (4)-(6) above for $r=-2,-3$, we have:

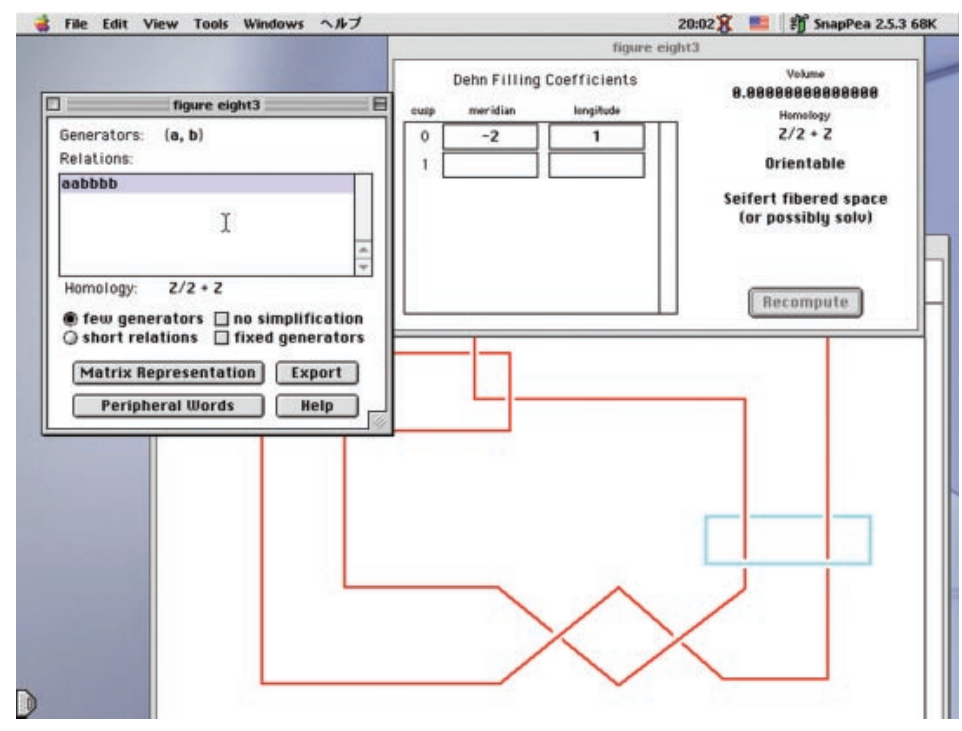

Picture 5.7.

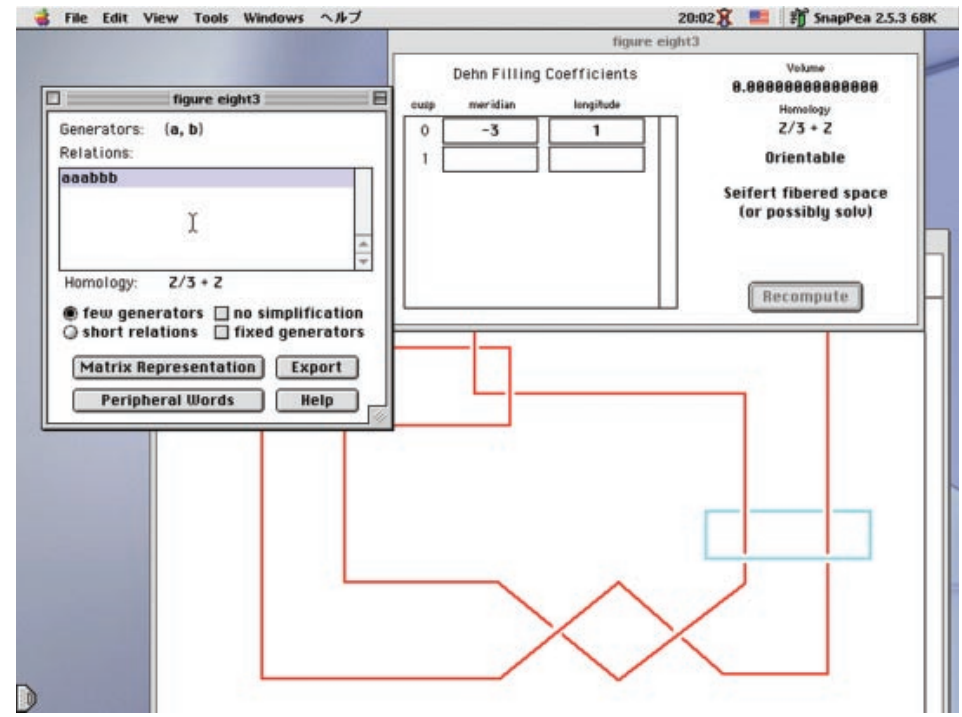

Picture 5.8.

These suggest that $c_{1}$ becomes an exceptional fiber of index 5 in $(K ;-2)$, and that $c_{1}$ becomes an exceptional fiber of index 4 in $(K ;-3)$.

Now we give a proper proof of: 
Proposition 4.3. For the figure-eight knot $K,(K ;-1)$ is a Seifert fiber space over $S^{2}$ with three exceptional fibers of indices 2, 3 and 7, and the trivial knot $c_{1}$ in Fig. 4.1 becomes an exceptional fiber of index 7.

Proof of Proposition 4.3. The sequence in Fig. 4.2 shows that $\left(K^{\prime} ; 1\right) \cong(K ;-1)$ where $K^{\prime}$ is the left handed trefoil. Follow the sequence backward. We then see that $c_{1}$, a trivial knot in $S^{3}$ disjoint from $K$ [Fig. 4.2(d)], can be regarded as a meridian of $K^{\prime}$ when identifying $(K ;-1)$ with $\left(K^{\prime} ; 1\right)$ [Fig. 4.2(a)].

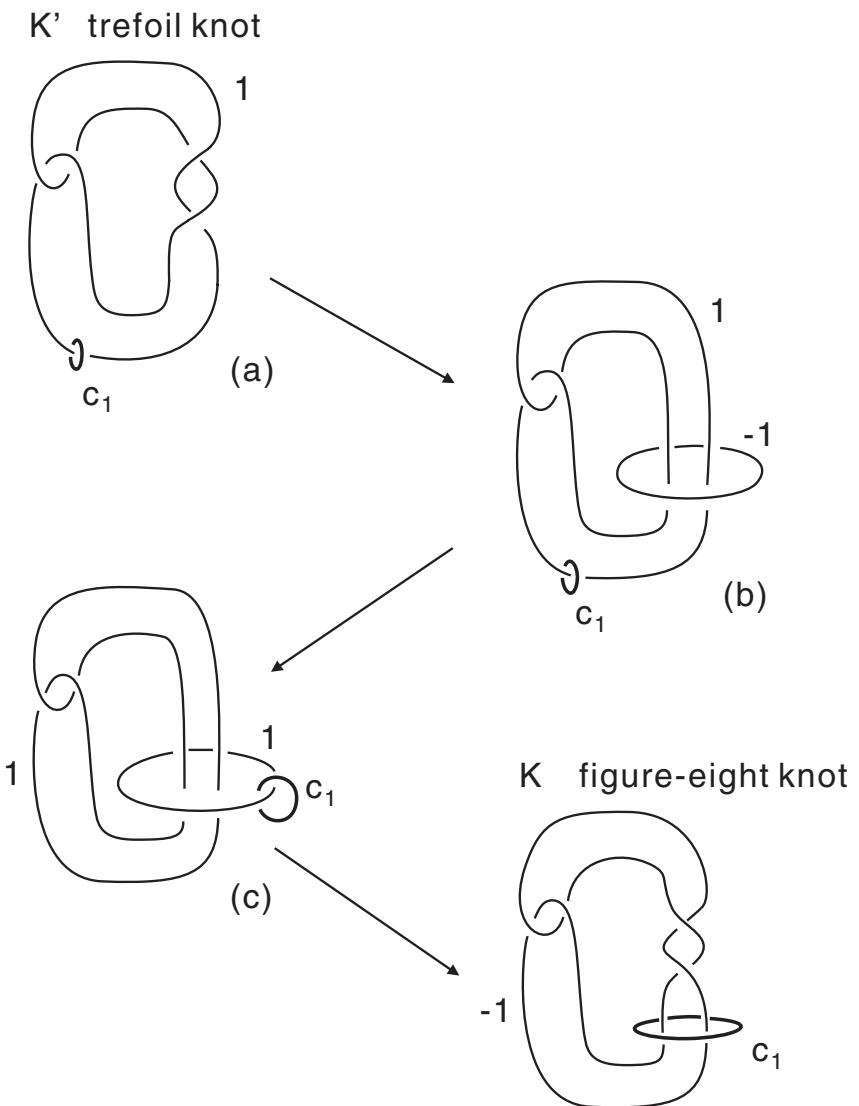

(d)

Fig. 4.2.

Recall that $E\left(K^{\prime}\right)$ is a Seifert fiber space over the disk with two exceptional fibers of indices 2, 3, and that the fiber slope on $\partial E\left(K^{\prime}\right)$ is -6 . Since the minimal geometric intersection number of the fiber slope -6 and the surgery slope 1 is 7, we can extend the Seifert fibration of $E\left(K^{\prime}\right)$ to that of $\left(K^{\prime} ; 1\right)$ so that the core of the reglued solid torus is an exceptional fiber of index 7. Thus $\left(K^{\prime} ; 1\right)$ is a Seifert fiber space over $S^{2}$ with three exceptional fibers of indices 2,3 and 7. Furthermore, since the surgery slope on $K^{\prime}$ is an integer, a meridian of $K^{\prime}$ is a longitude of the reglued solid torus. This implies that a meridian of $K^{\prime}$ is isotopic to the core of the reglued solid torus in $\left(K^{\prime} ; 1\right)$, i.e., the exceptional fiber of index 7.

Hence, after isotoping the fibration slightly, the trivial knot $c_{1} \subset S^{3}$ disjoint from $K$ becomes an exceptional fiber of index 7 in $(K ;-1)$.

Next we consider $c_{2}$ instead of $c_{1}$.

\section{SnapPea Experiment 6 (Seifert fibered surgeries on the figure-eight knot II)}

(1) Applying the same method in SnapPea Experiment 5, we have Pictures 6.1, 6.2 and 6.3.

These suggest that $c_{2}$ becomes an exceptional fiber of index 7 in $(K ;-1)$, an exceptional fiber of index 4 in $(K ;-2)$ and an exceptional fiber of index 3 in $(K ;-3)$.

Here we prove these facts only in the case where $r=-1$.

Proposition 4.4. For the figure-eight knot $K,(K ;-1)$ is a Seifert fiber space over $S^{2}$ with three exceptional fibers of indices 2, 3 and 7 and the trivial knot $c_{2}$ in Fig. 4.1 becomes an exceptional fiber of index 7. 


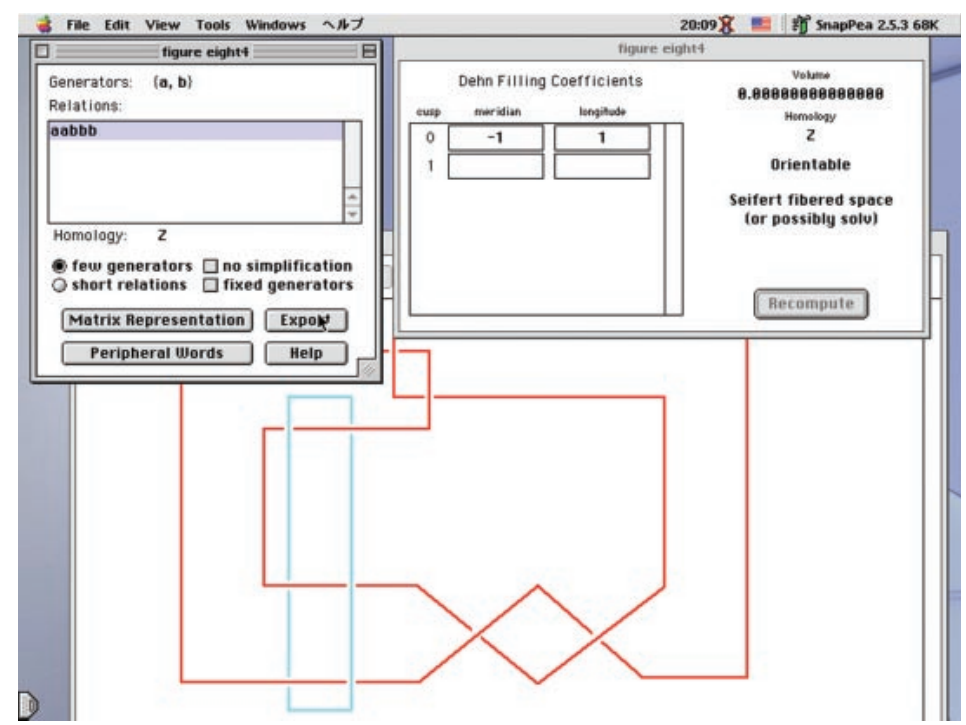

Picture 6.1.

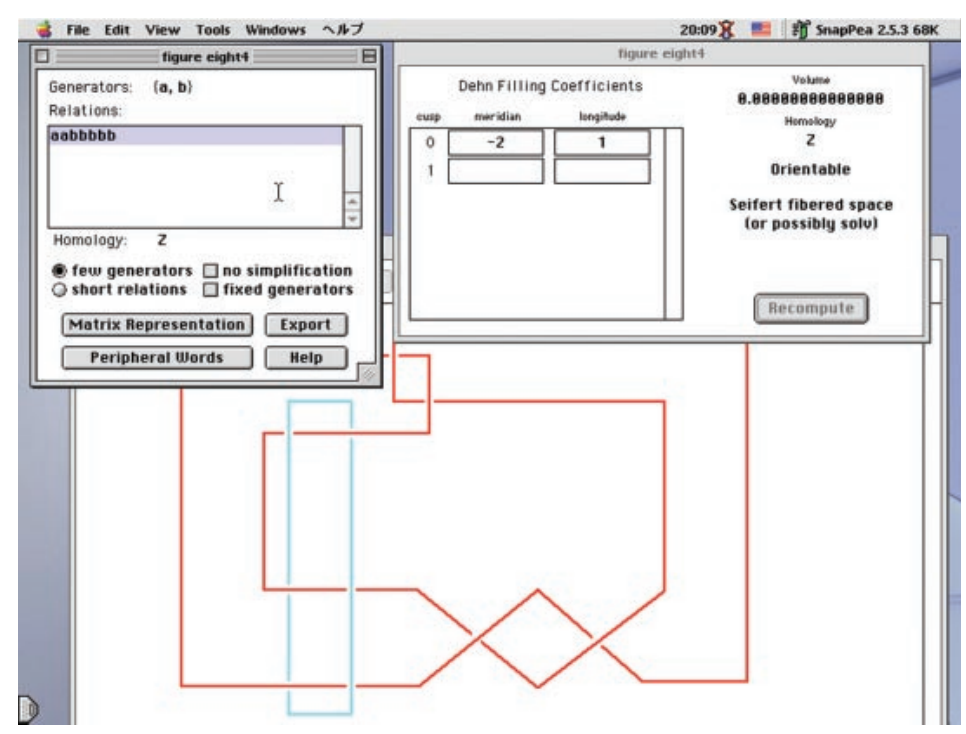

Picture 6.2.

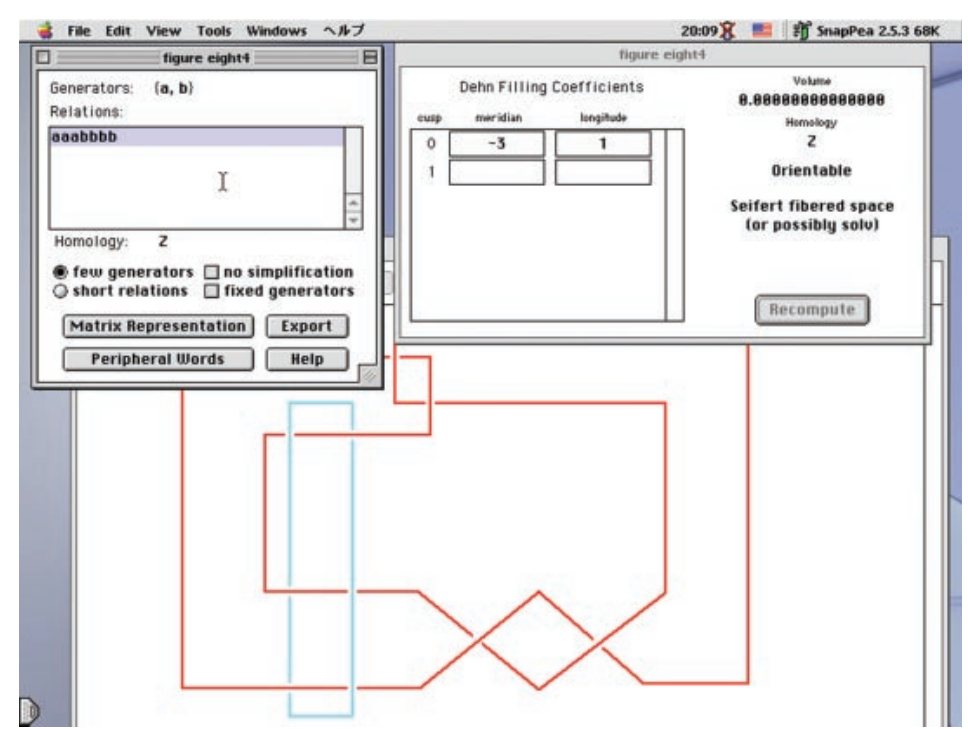

Picture 6.3. 
Proof of Proposition 4.4. Consider the sequence in Fig. 4.3 and apply a similar argument in the proof of Proposition 4.3.

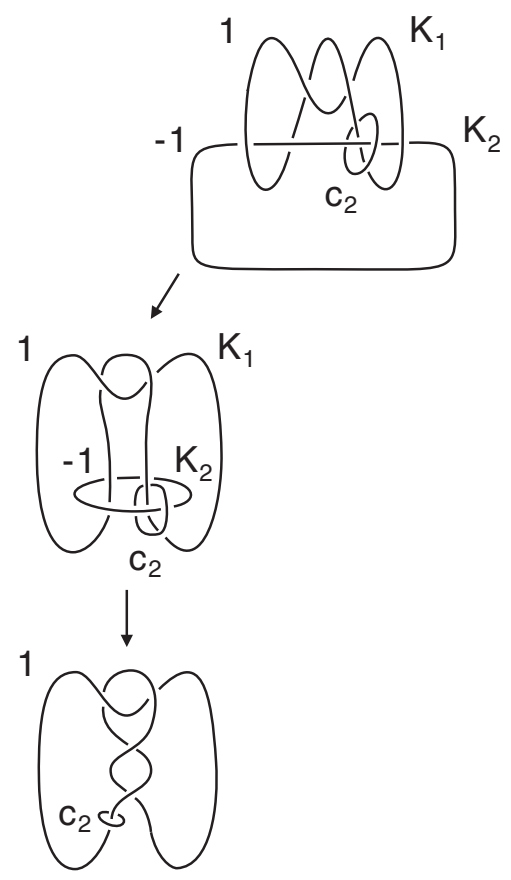

$\mathrm{K}_{1}^{\prime}$ trefoil knot
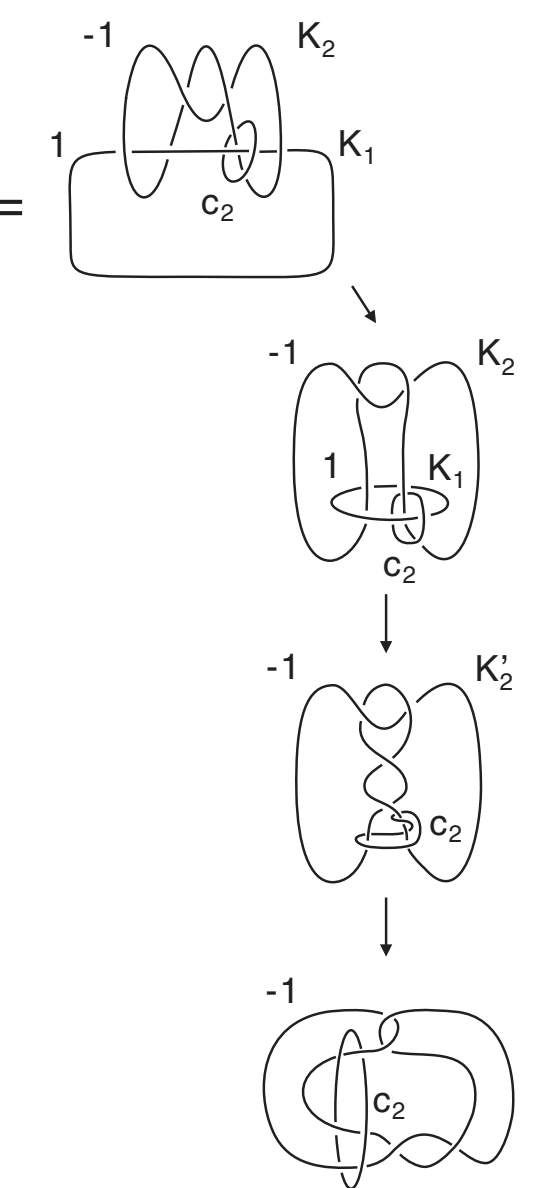

$\mathrm{K}_{2}$ figure-eight knot

Fig. 4.3.

To sum up we have:

\begin{tabular}{|c|c|c|c|c|}
\hline & $S^{3}$ & $(K ;-1)$ & $(K ;-2)$ & $(K ;-3)$ \\
\hline$c_{1}$ & unknot & fiber of index 7 & fiber of index 5 & fiber of index 4 \\
$c_{2}$ & unknot & fiber of index 7 & fiber of index 4 & fiber of index 3 \\
\hline
\end{tabular}

These examples lead us to the following conjecture, which answers Question 1.

Conjecture 4.5 ([22]). Let $K$ be a knot in $S^{3}$. If $(K ; r)$ is a Seifert fiber space, then it admits a Seifert fibration such that a fiber of it is unknotted in (the original) $S^{3}$.

In [22, Section 6] we verified this conjecture for some Seifert fibered surgeries on the following knots:

- 2-bridge knots,

- Eudave-Muñoz' hyperbolic knots with non-hyperbolic surgeries [6], and

- Some twisted torus knots.

Recently, in [7], Eudave-Muñoz introduced new infinite families of hyperbolic knots admitting Seifert fibered surgeries and verified Conjecture 4.5 for these families. More recently, Mattman, Miyazaki and myself [19] discovered infinite families of knots each of which admits a Seifert fibered surgery with none of these surgeries coming from Dean's primitive/Seifert-fibered construction, for the definition of primitive/Seifert-fibered construction, see [5, 10]. These families give a negative answer to [10, Conjecture 4.6]. However these Seifert fibered surgeries satisfy Conjecture 4.5 .

Here is another important conjecture which is still open, see [10]. 
Conjecture 4.6. If $(K ; r)\left(r \neq \frac{1}{0}\right)$ is a Seifert fiber space, then either $r$ is an integer, or $K$ is a trivial knot, a torus knot or a cable of a torus knot.

In [22], we proved:

Theorem 4.7 ([22]). Suppose that $(K ; r)\left(r \neq \frac{1}{0}\right)$ is a Seifert fiber space. If the surgery satisfies Conjecture 4.5, then it satisfies Conjecture 4.6.

Remark. Hayashi [12] and Hayashi-Motegi [13] also obtained some weaker results under some assumption of the position of fibers.

\section{Closed Geodesics and Seifert Fibers}

Let $K$ be a hyperbolic knot in $S^{3}$. Suppose that the hyperbolic structure of $S^{3}-K$ degenerates to a Seifert fibering structure of $(K ; r)$. In the previous section we consider the question: Which curves in $S^{3}-K$ become fibers in $(K ; r)$ ? Conjecture 4.5 states that we can always find a trivial knot as the candidates for such curves. Experiments via SnapPea suggest that some closed geodesics in the hyperbolic manifold $S^{3}-K$ can be candidates for such curves.

We start again with Seifert fibered surgeries on the figure-eight knot $K$. Let $c_{1}$ and $c_{2}$ be the trivial knots in $S^{3}-K$ depicted in Fig. 4.1.

\section{SnapPea Experiment 7 ( $c_{1}$ is the shortest closed geodesic)}

Let us check that $c_{1}$ is a shortest closed geodesic in the complement of the figure-eight knot.

(1) Following SnapPea Experiment 5 (1), (2), we have Picture 5.2, in which SnapPea shows that the complement of the figure-eight knot is hyperbolic.

(2) To get a list of closed geodesics, choose Length Spectrum in View menu (Picture 7.1).

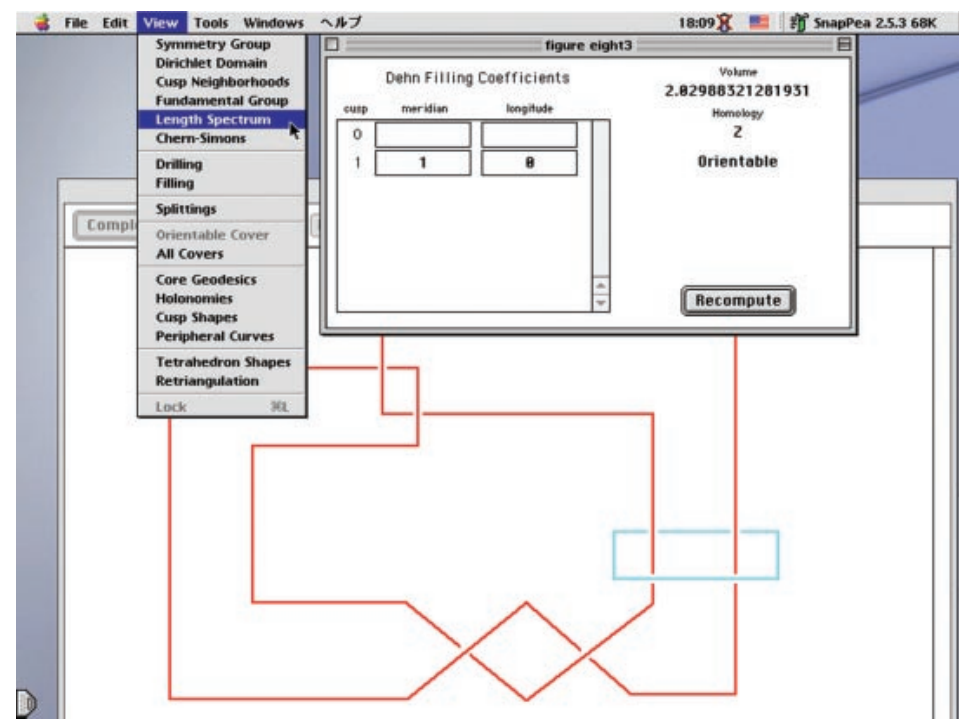

Picture 7.1.

Then we have the "Length Spectrum" window as in Picture 7.2. The important parameter is the "Find geodesics to length" box. SnapPea will compute and report all geodesics up to the cutoff length we specify here. In Picture 7.2, we put the number 2 in the box so that SnapPea finds geodesics up to length 2 in the complement $S^{3}-K$.

By clicking Compute button, SnapPea provides us the list of closed geodesics up to length $\leq 2$ (Picture 7.3). The table in the "Length Spectrum" window shows that there are two shortest closed geodesics having the same length $1.087 \ldots$ and distinct torsions $1.722 \ldots$ and $-1.722 \ldots$. (These closed geodesics are related by an orientation reversing isometry of $S^{3}-K$.)

(3) We choose Core Geodesics in View menu (Picture 7.4).

Then SnapPea shows that the $c_{1}$ has length $1.087 \ldots$ (Picture 7.5). Referring the list in the "Length Spectrum" window, we see that $c_{1}$ is a shortest geodesic in $S^{3}-K$. 


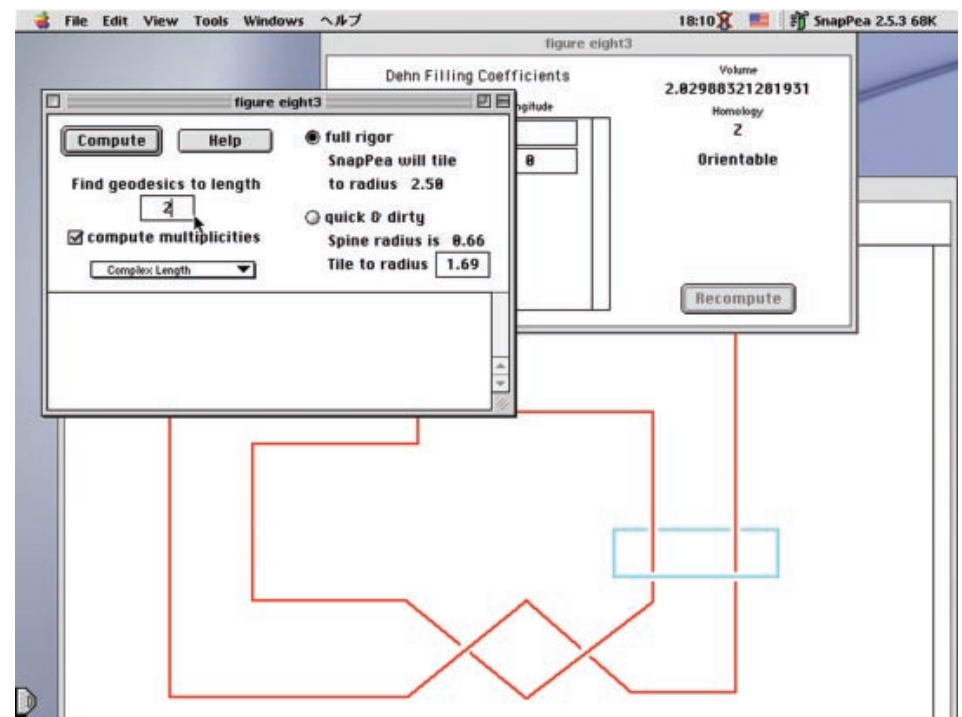

Picture 7.2.

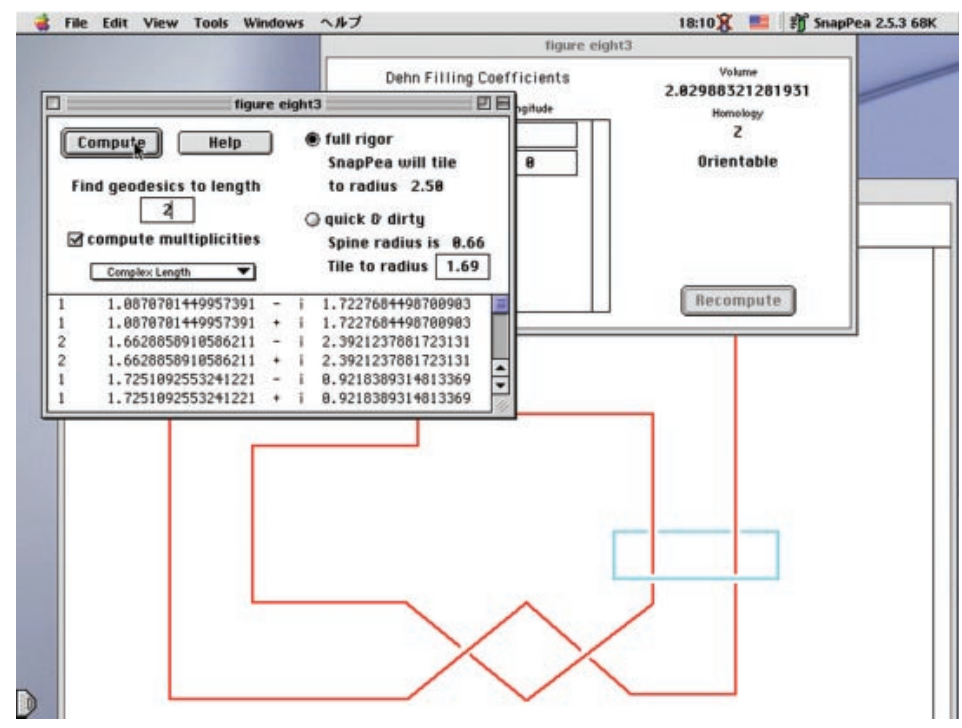

Picture 7.3.

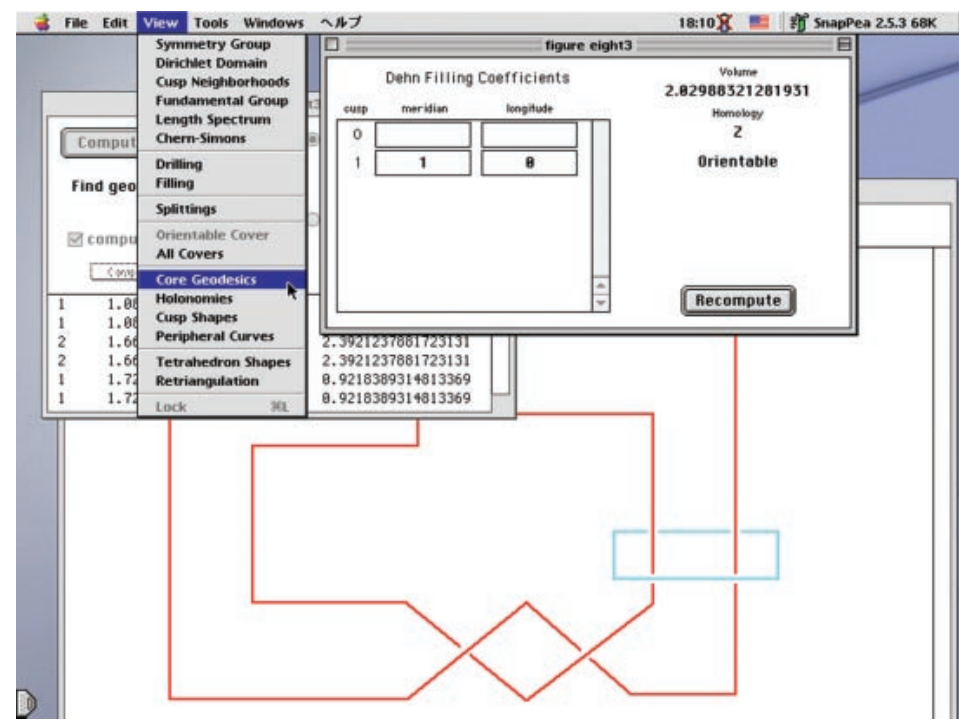

Picture 7.4. 


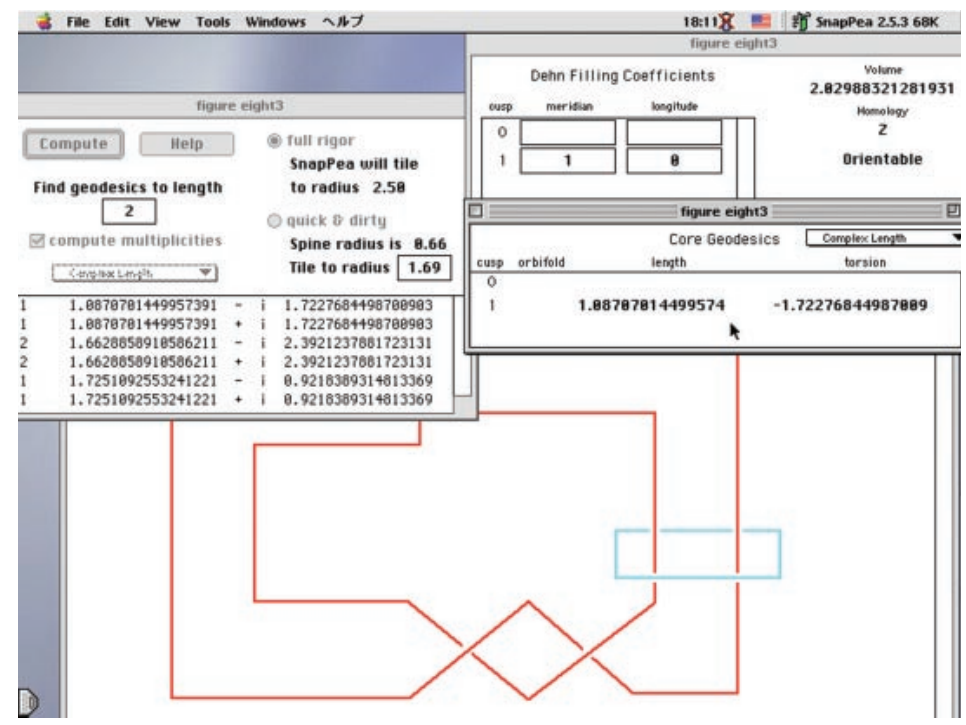

Picture 7.5

\section{SnapPea Experiment 8 ( $c_{2}$ is the second shortest closed geodesic)}

Applying the same procedures in SnapPea Experiment 7, we have Picture 8.1.

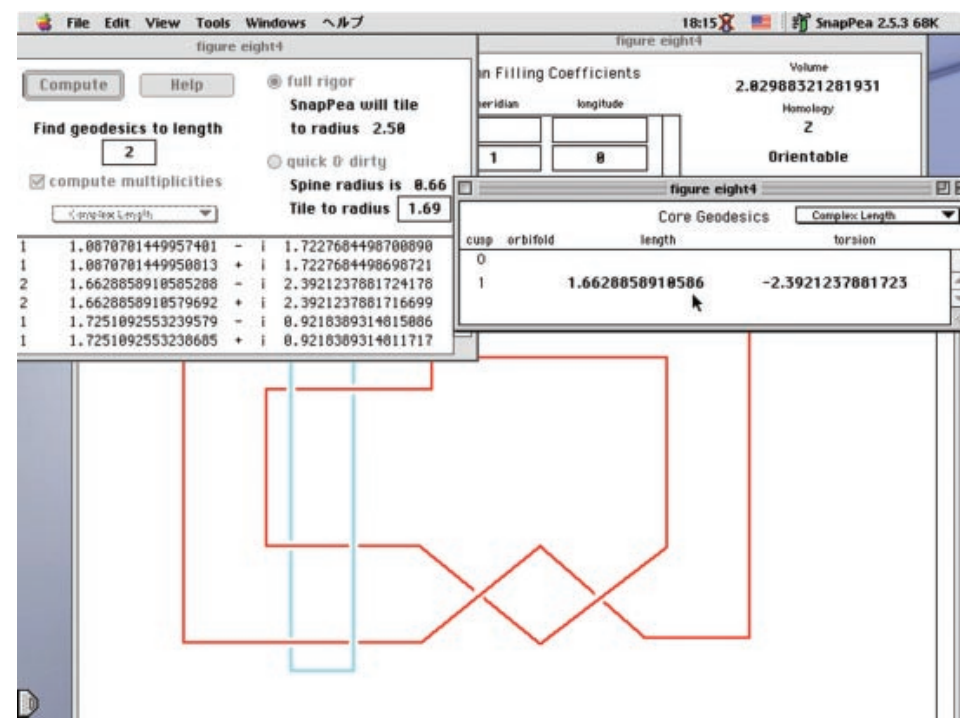

Picture 8.1.

This shows that $c_{2}$ is the second shortest geodesic in the complement of the figure-eight knot.

Remark. Recently Miller [20] studies geodesic knots in the figure knot complement in detail.

SnapPea Experiments 7 and 8 suggest that short closed geodesics in hyperbolic knot complements often serve as $c$ in Question 1. We give more examples of this kind.

Example (twist knots). Let $K_{2,2 n}$ be the twist knot and $c$ the trivial knot described in Fig. 5.1.

It is known that $K_{2,2 n}$ is hyperbolic for $n \neq 0,1$. As shown in [22, Proposition 6.1], $\left(K_{2,2 n} ; r\right)$ is a Seifert fiber space with $c$ an exceptional fiber in $\left(K_{2,2 n} ; r\right)$, where $r=-1,-2,-3$. Note that $K_{2,0} \cup c$ is the Whitehead link, and $K_{2,2 n}$ is obtained from $K_{2,0}$ by performing $-1 / n$-surgery on $c$. From Thurston's hyperbolic Dehn surgery theorem [27, 28], $c$ is the unique shortest geodesic in $S^{3}-K_{2,2 n}$ if $|n|$ is sufficiently large, and the length of $c$ tends to 0 as $|n| \rightarrow \infty$. This result, together with tests by SnapPea for small $n$, suggests that $c$ is the shortest geodesic in $S^{3}-K_{2,2 n}$ for any $n \neq 0,1$.

\section{SnapPea Experiment 9 (Seifert fibered surgeries on twist knots)}

Here we apply the method which was given in Sect. 3 to check that $c$ in Fig. 5.1 is a shortest closed geodesic in 


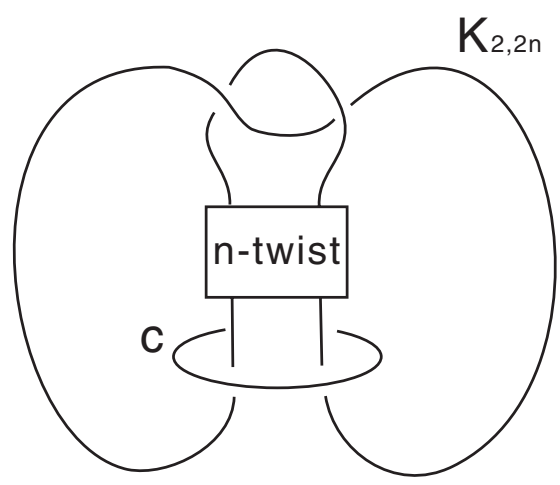

Fig. 5.1.

$S^{3}-K_{2,10}$ and $c$ becomes a fiber in $\left(K_{2,10} ;-3\right)$. Note that $K_{2,10}$ is obtained from $K_{2,0}$ by 5 -twisting about $c$.

(1) Draw a diagram of the Whitehead link $K_{2,0} \cup c$ and fill in the meridian-longitude boxes in the second row with 1 and -5 respectively to obtain $K_{2,10}$, see SnapPea Experiment 3. After clicking Recompute button, we have Picture 9.1, in which SnapPea shows that $K_{2,10}$ is a hyperbolic knot with hyperbolic volume $3.553 \ldots$.

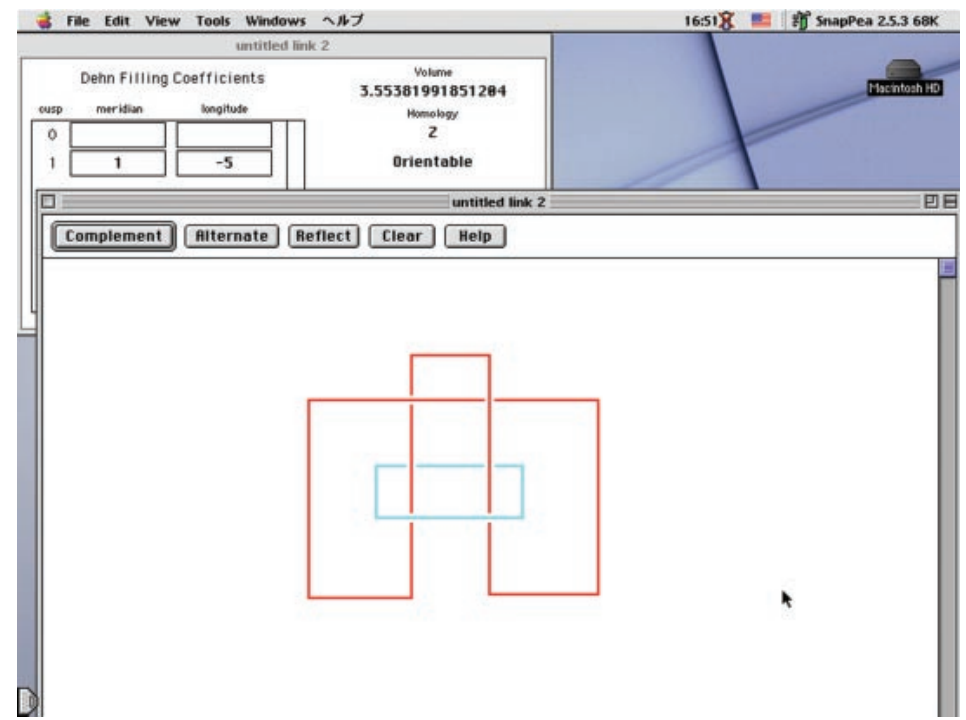

Picture 9.1.

(2) Apply the procedure as in SnapPea Experiment 7 (2), we have Picture 9.2, in which SnapPea shows that there is a unique shortest closed geodesic of length $0.070 \ldots$ in $S^{3}-K_{2,10}$.

(3) Choose Core Geodesics in View menu, we have Picture 9.3, in which SnapPea shows that $c$ is the shortest closed geodesic in $S^{3}-K_{2,10}$. (Compare the length of the shortest geodesic in the "Length Spectrum" window and the length of the core geodesic.)

Let us check that $c$ becomes a fiber in the Seifert fiber space $\left(K_{2,10} ;-3\right)$. To recognize that $\left(K_{2,10} ;-3\right)$ is, in fact, Seifert fibered, we need a proper proof as in [3], see also [22]. Here we appply SnapPea Experiment 4 to observe that $\left(K_{2,10} ;-3\right)$ is non-hyperbolic.

(4) Note that the linking number of $K_{2,0}$ (the red component) and $c$ (the blue component) is zero. The correct surgery coefficient $\frac{p}{q}=-3-5 \times 0^{2}=-3$, see Proposition 3.1. Thus to perform -3 -surgery on $K_{2,10}$, we fill in the meridian-longitude boxes in the first row with -3 and 1 respectively as in Picture 9.4. Clicking Recompute button, we have a message suggesting that the result is not hyperbolic.

(5) Following the procedures in SnapPea Experiment 5 (4)-(6), we have Picture 9.5.

This suggests that $c$ becomes a fiber in $\left(K_{2,10} ;-3\right)$.

Exercise. Get a feel that the length of $c$ in $S^{3}-K_{2,2 n}$ tends to 0 as $|n| \rightarrow \infty$ by applying SnapPea Experiment 9 (1) and (3).

SnapPea Experiments 7 and 9 lead us to more specific question. 


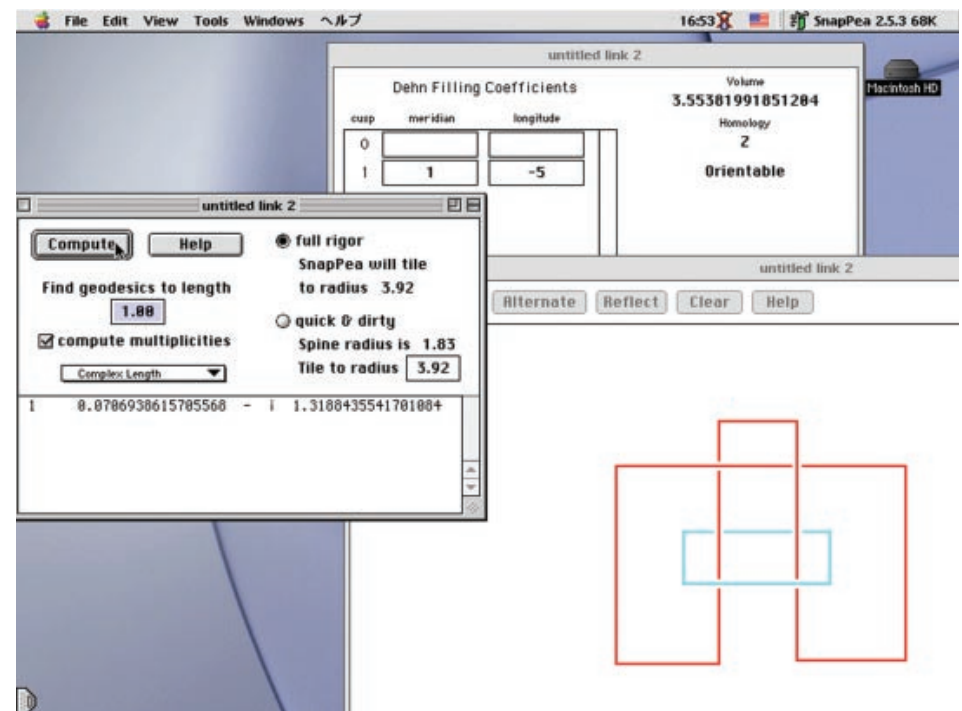

Picture 9.2.

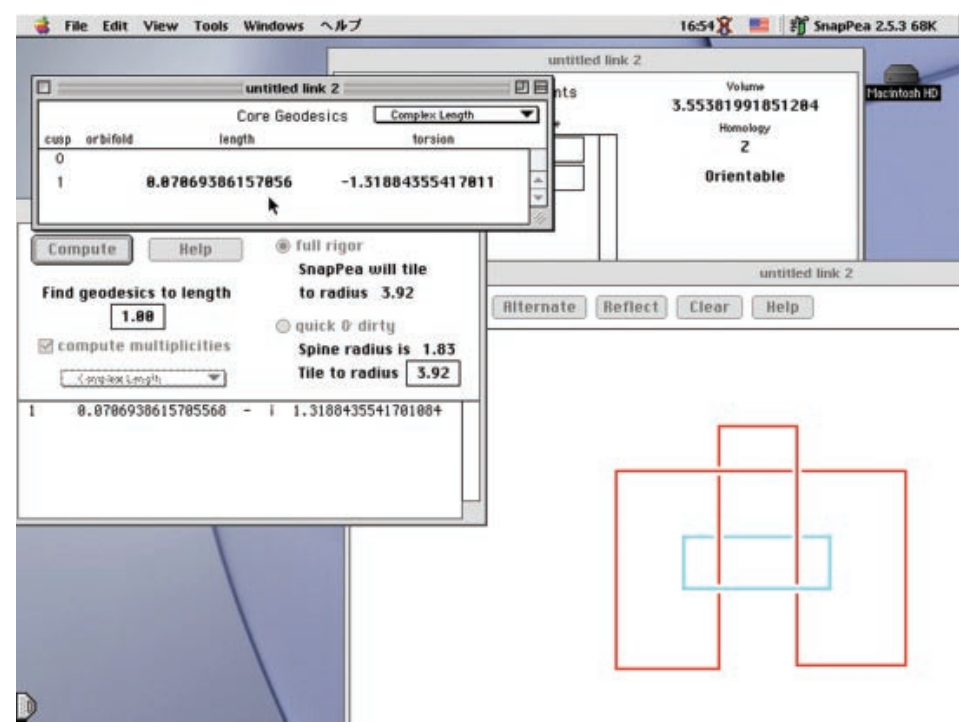

Picture 9.3.

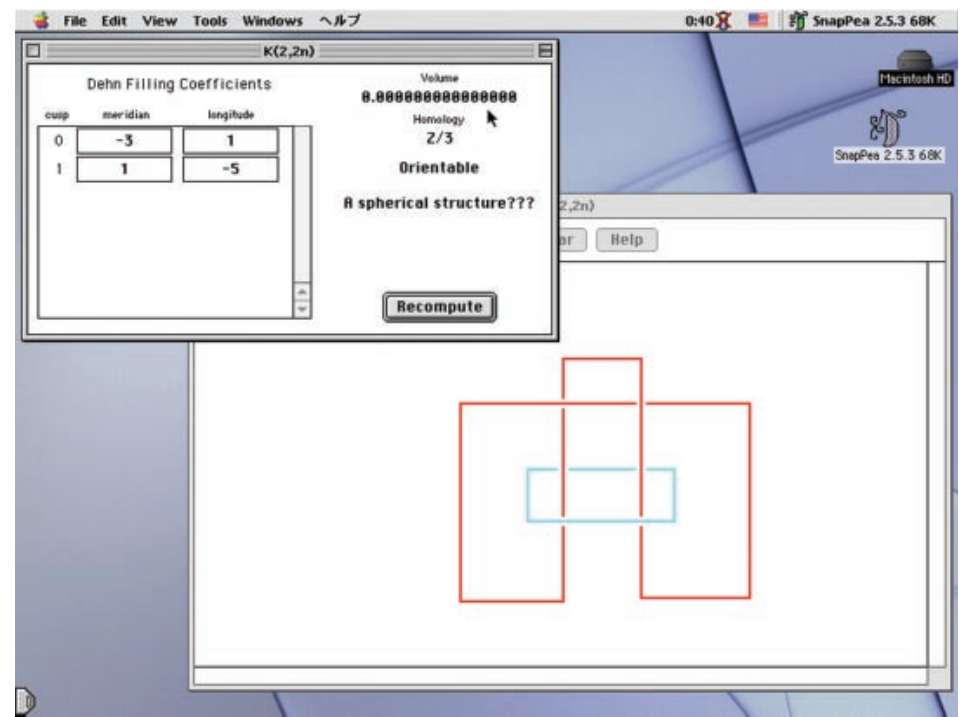

Picture 9.4 


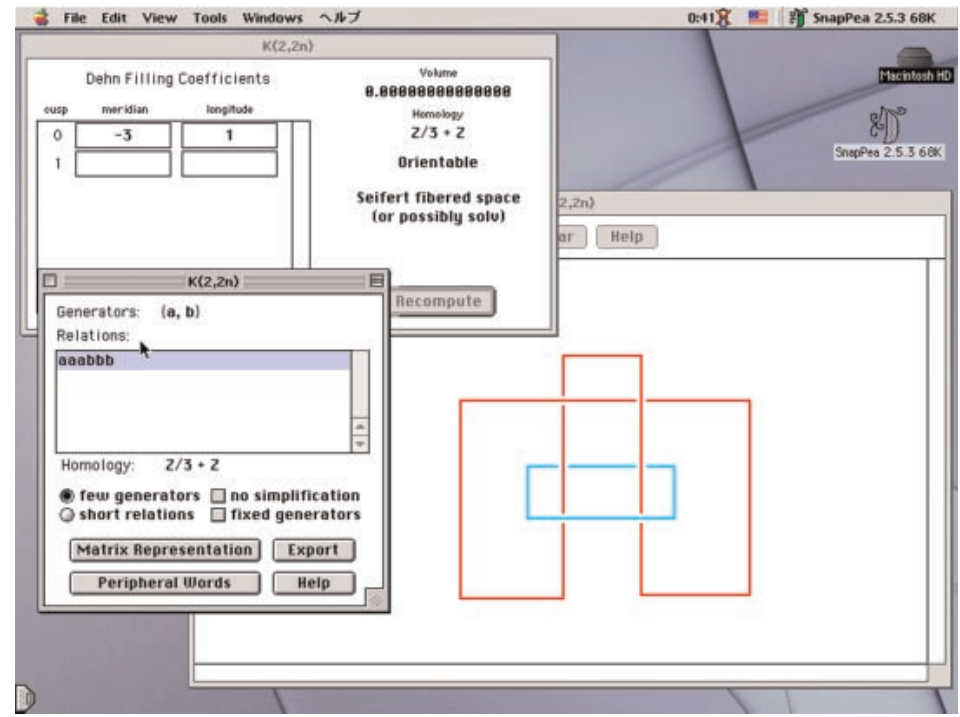

Picture 9.5.

Question 2. Suppose that $K$ is a hyperbolic knot in $S^{3}$, and $(K ; r)$ is Seifert fibered. Then does a "shortest closed geodesic" c in $S^{3}-K$ satisfy that:

- $c$ is a trivial knot in $S^{3}$, and

- $c$ is a fiber in some Seifert fibration of $(K ; r)$ ?

In [22, Example 3], we investigated surgeries on Eudave-Muñoz' hyperbolic knots and gave another supporting evidence of the positive answer to this question. However, the following example demonstrates that Question 2 is negative in general. It should be mentioned that in the example, instead of the shortest closed geodesic, the second shortest one satisfies the condition in Question 2.

\section{SnapPea Experiment 10 (Seifert fibered surgery on a twisted torus knot)}

Consider a link depicted in Picture 10.1; the red component $K$ is a $(3,7)$-torus knot. Let $K_{-1}$ be a knot obtained from $K$ by (-1)-twisting about the blue component $c$. The linking number of $K$ and $c$ is 10 . The knot $K_{-1}$ is referred to as the twisted torus knot $K(3,7,10,-1)$ [22] and [23], see also [5] for twisted torus knots of distinct types.

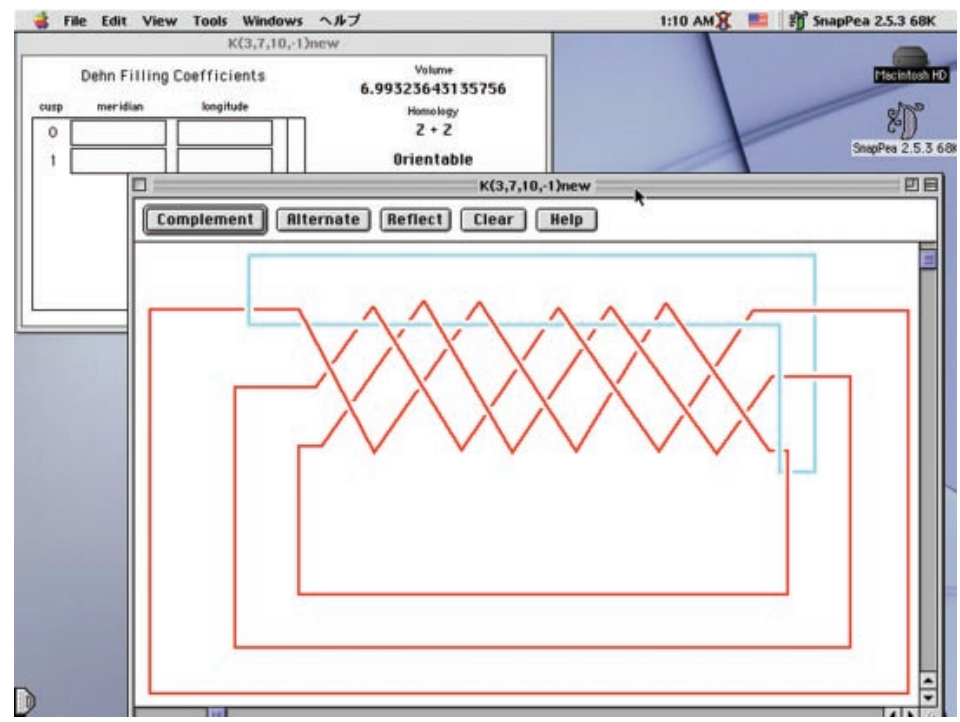

Picture 10.1.

(1) To obtain the twisted torus knot $K_{-1}=K(3,7,10,-1)$ using the method in Sect. 3, we perform 1-surgery on $c$ and click Recompute button, see Picture 10.2.

SnapPea shows that the twisted torus knot $K(3,7,10,-1)$ is a hyperbolic knot with hyperbolic volume $6.128 \ldots$ In fact, applying the argument in [23], we can show that $K_{-1}=K(3,7,10,-1)$ is hyperbolic. 


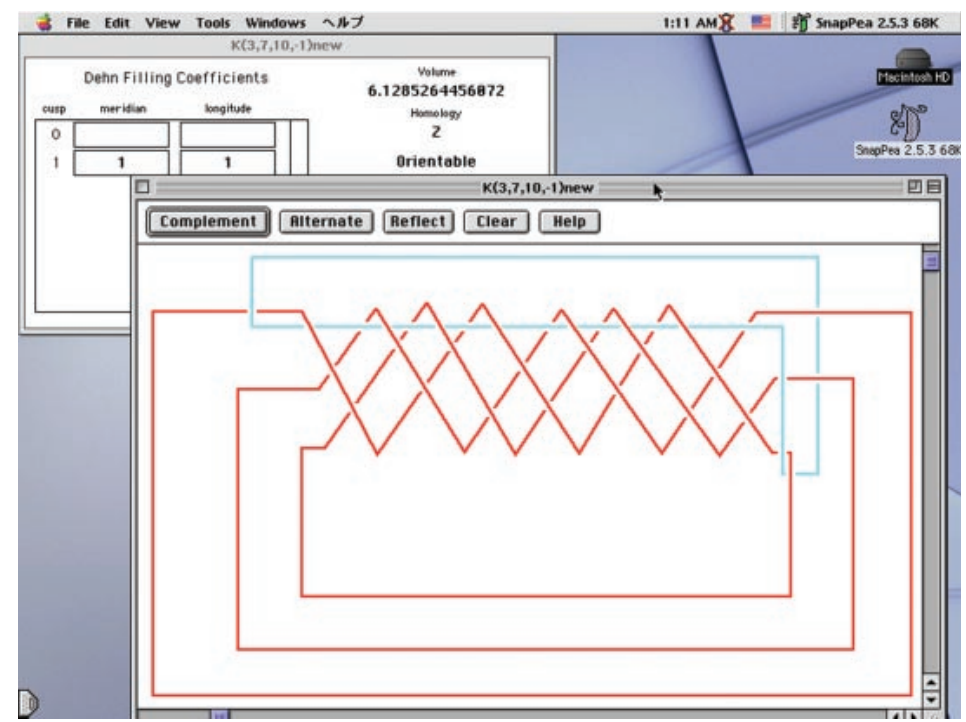

Picture 10.2.

(2) Perform -79-surgery on $K_{-1}$. Since the linking number of $K$ and $c$ is 10 , the correct surgery coefficient $\frac{p}{q}=-79-(-1) \times 10^{2}=21$ (Proposition 3.1). Hence to perform -79 -surgery on $K_{-1}$, we fill in the meridianlongitude boxes in the first row with 21 and 1 respectively as in Picture 10.3. Clicking Recompute button to get an information about $\left(K_{-1} ;-79\right)$, we have a message suggesting that the result is not hyperbolic.

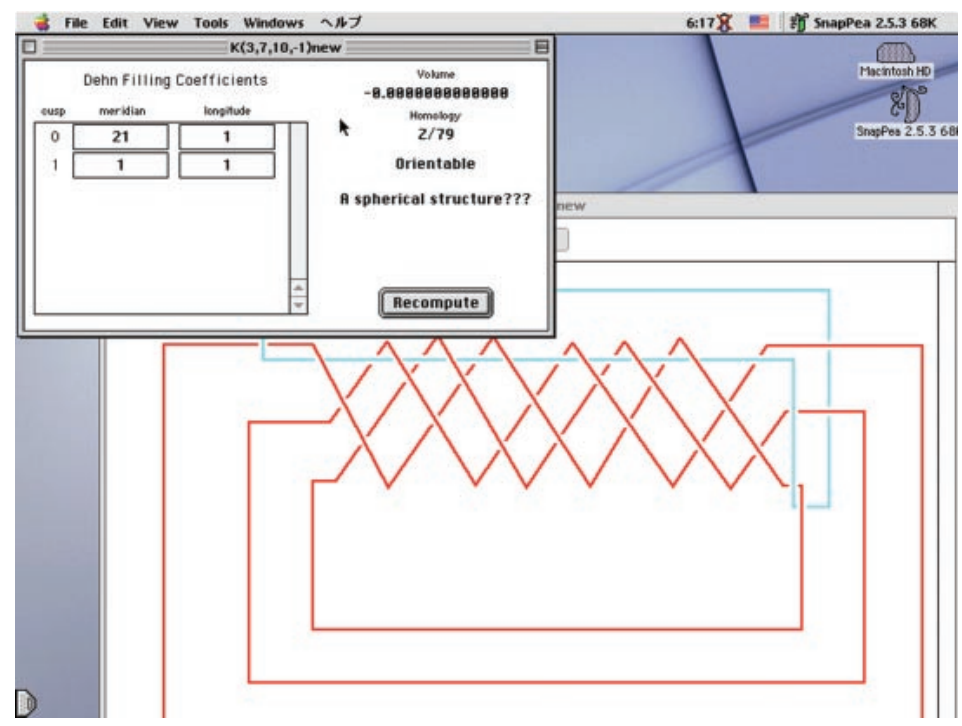

Picture 10.3.

Moreover, by choosing Fundamental Group in View menu, we have Picture 10.4 in which SnapPea shows that $\pi_{1}\left(\left(K_{-1} ;-79\right)\right) \cong \mathbb{Z}_{79}$. This then suggests that $\left(K_{-1} ;-79\right)$ is a lens space.

For a proper proof of this, see [22, Proposition 6.4].

(3) As in SnapPea Experiment 7 (2), we have Picture 10.5 in which SnapPea reports all geodesics up to length 1.

(4) To drill out the shortest geodesic, choose Drilling in View menu (Picture 10.6).

(5) Then we have the "Drilling" window which offers a selection of closed geodesics. We select the shortest closed geodesic in the window by clicking (Picture 10.7) and then click Drill button (Picture 10.8).

Then we have a new "Dehn filling" window in which the new cusp is added to the end of the manifold's cusp list (Picture 10.9)

(6) To check whether the curve we have drilled is isotopic to the intended geodesic, perform a $\frac{1}{0}$-Dehn filling to restore the original hyperbolic structure (Picture 10.10).

(7) Consider the complement $S^{3}-$ (shortest closed geodesic), which can be obtained as $\left(K_{-1} ; \frac{1}{0}\right)-($ third component). To get this manifold we fill in the meridian-longitude boxes in the first row with 1 and 0 


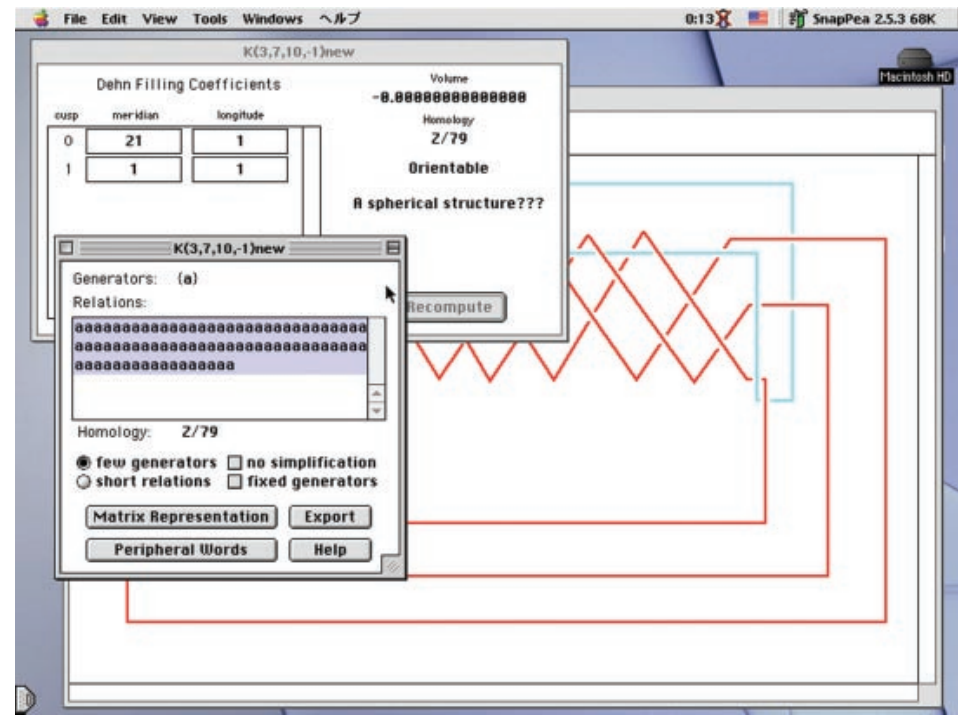

Picture 10.4.

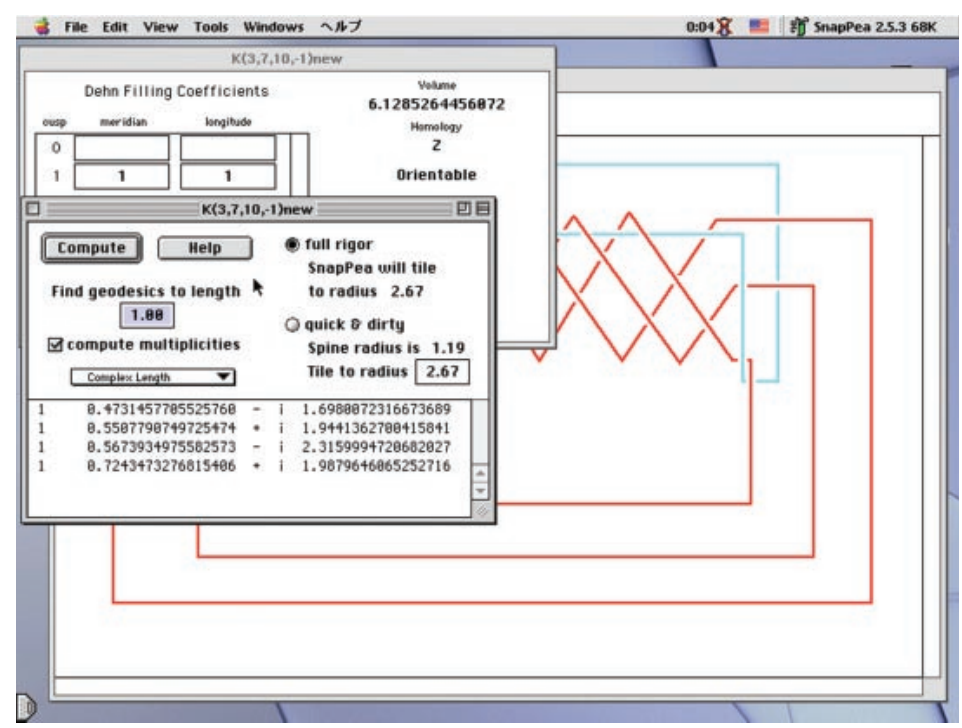

Picture 10.5.

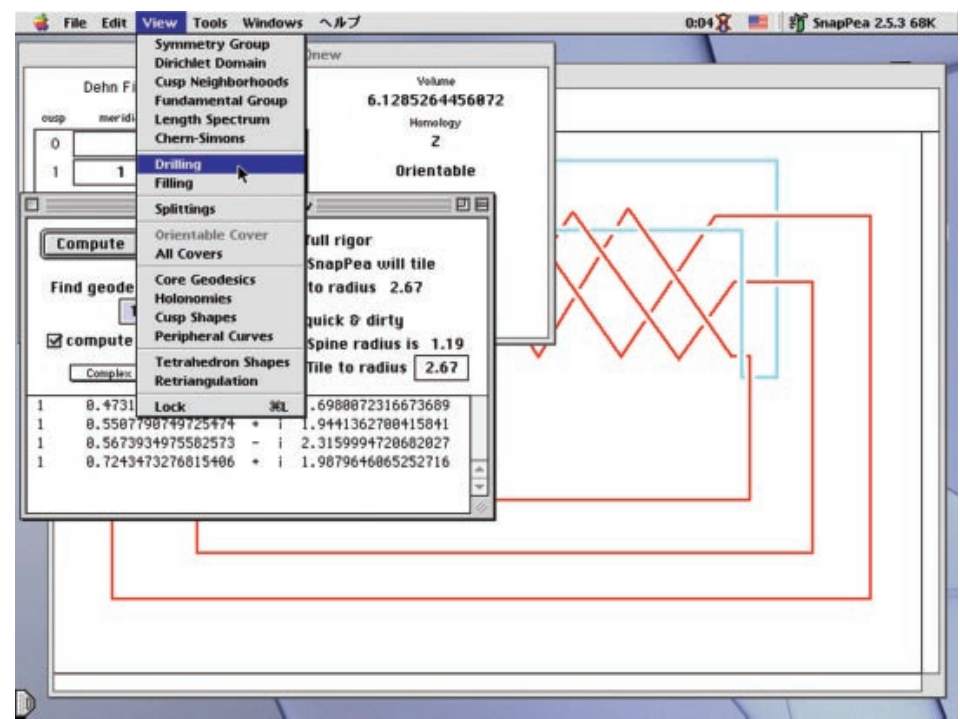

Picture 10.6. 


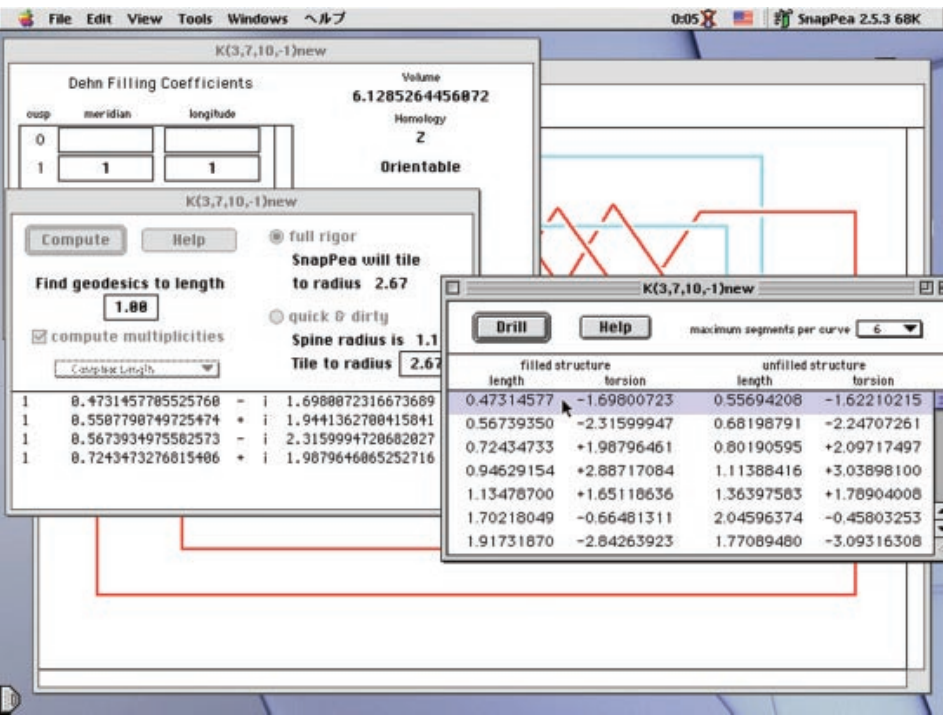

Picture 10.7

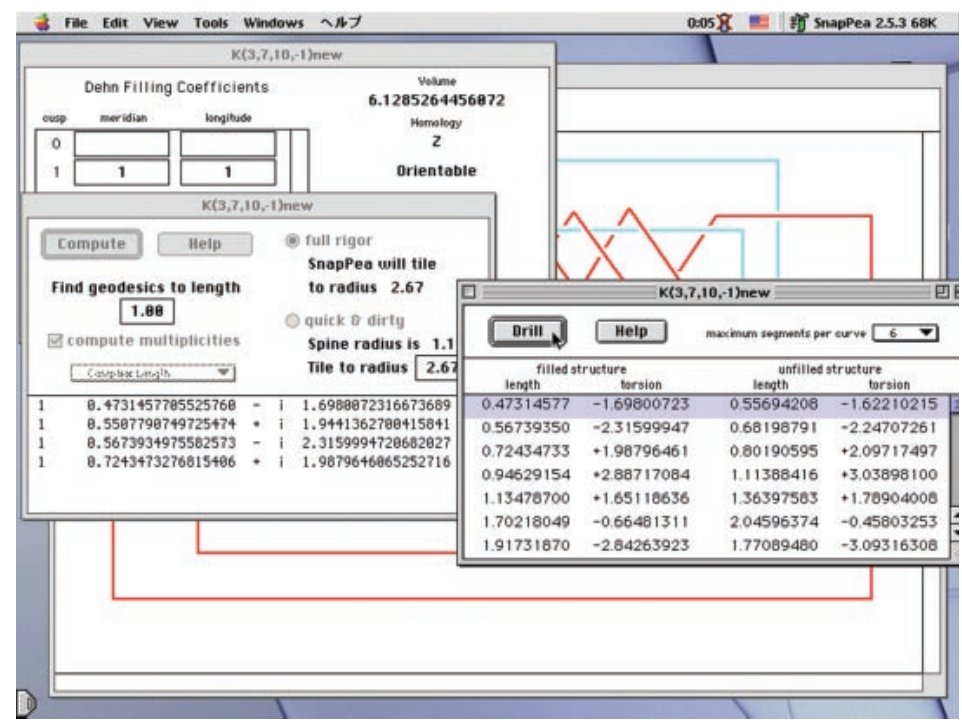

Picture 10.8 .

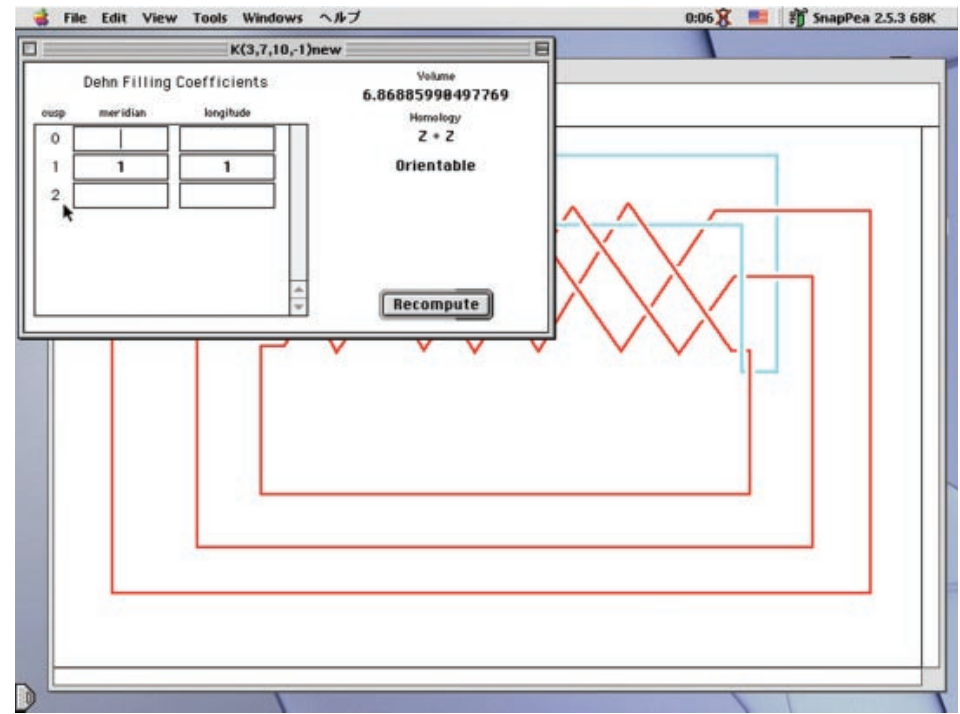

Picture 10.9 


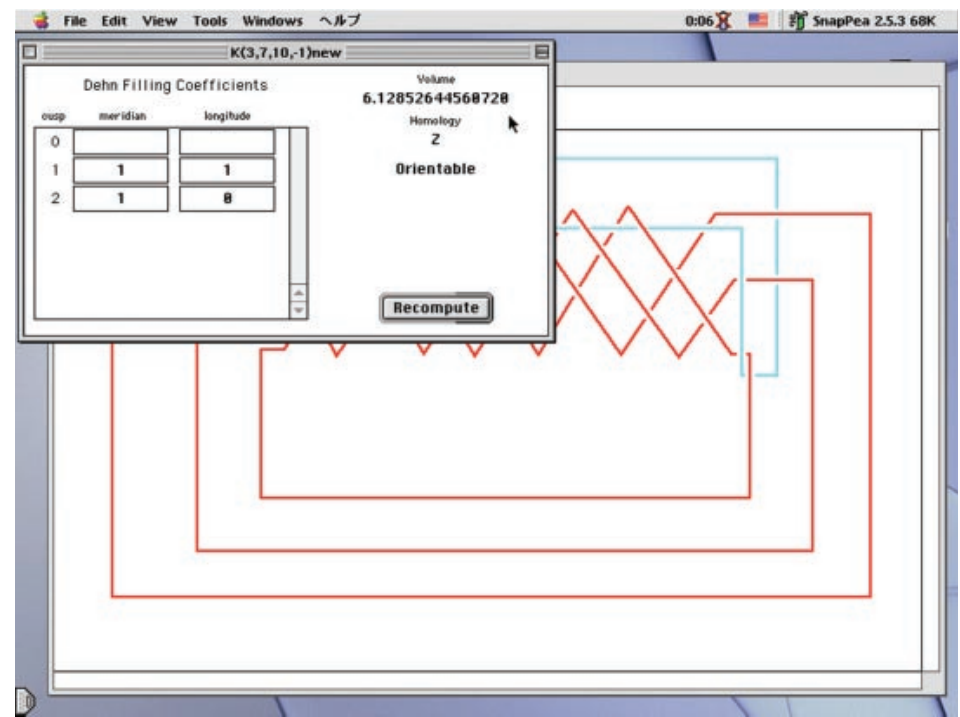

Picture 10.10 .

respectively (the meridian is not changed under twisting about $c$ ) and delete 1, 0 from the third row as in Picture 10.11. Clicking Recompute button, we have a message which suggests that the complement of the shortest closed geodesic in $S^{3}$ is not hyperbolic (Picture 10.11).

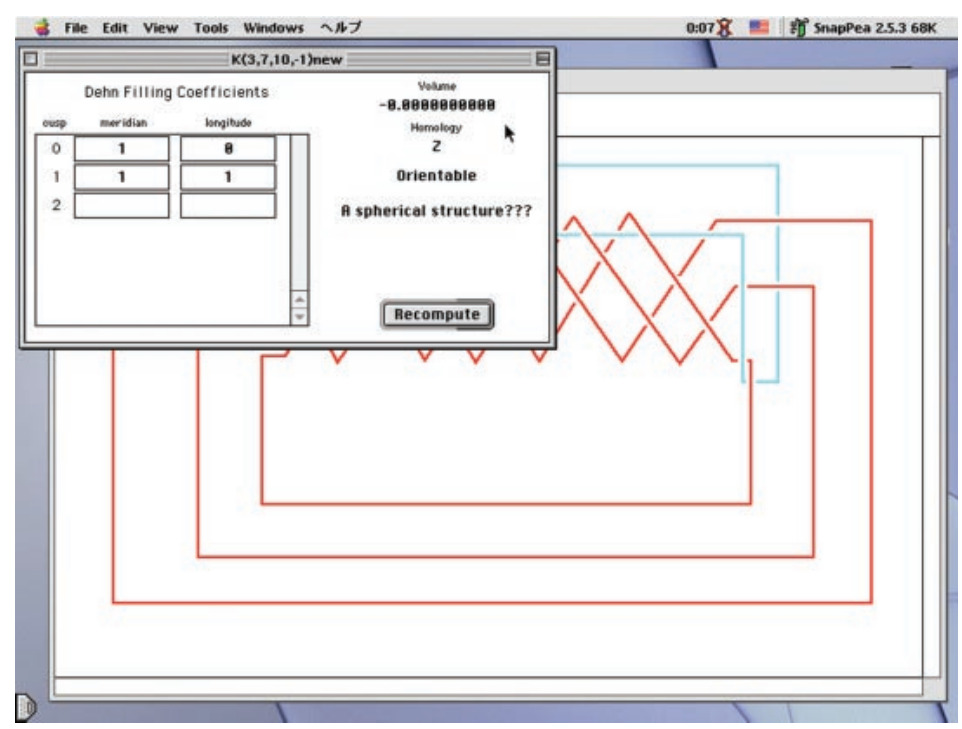

Picture 10.11.

(8) Check the fundamental group of the complement of the shortest closed geodesic in $S^{3}$. Choose Fundamental Group in View menu to get a presentation.

In Picture 10.12, SnapPea gives the presentation of $\pi_{1}\left(S^{3}-\right.$ (shortest closed geodesic)):

$$
\pi_{1}=\left\langle a, b \mid a^{2} b^{3}\right\rangle \text {. }
$$

Applying the argument in SnapPea Experiment 5 (6), we see that the exterior $S^{3}-$ int $N$ (shortest closed geodesic) is Seifert fibered with the above fundamental group, and hence that the shortest closed geodesic is a trefoil knot in $S^{3}$.

(9) Let us see that the shortest closed geodesic becomes a fiber in $\left(K_{-1} ;-79\right)$. Apply a similar method, we have Picture 10.13, in which SnapPea shows that $\pi_{1}\left(\left(K_{-1} ;-79\right)-\right.$ int $N($ shortest closed geodesic $\left.)\right) \cong \pi_{1}\left(\left(K_{-1}\right.\right.$; $-79)-($ shortest closed geodesic $)) \cong \mathbb{Z}$.

It turns out that $\left(K_{-1} ;-79\right)-$ int $N$ (shortest closed geodesic) is a solid torus and the shortest closed geodesic becomes a fiber in $\left(K_{-1} ;-79\right)$. More precisely, the shortest closed geodesic becomes a core of a genus one 


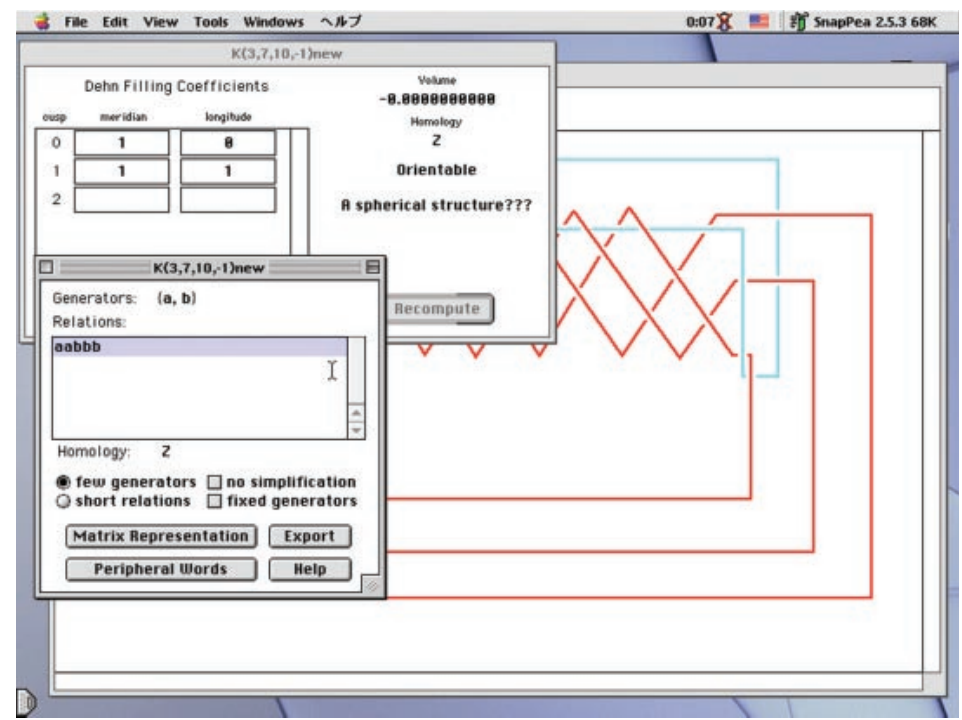

Picture 10.12.

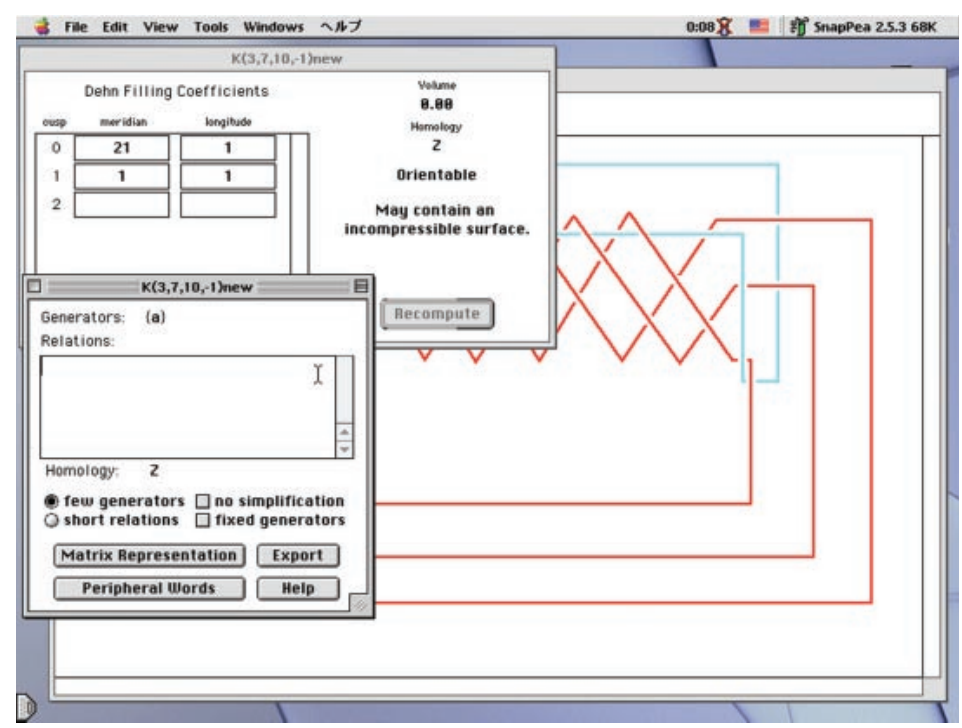

Picture 10.13.

Heegaard splitting of the lens space $\left(K_{-1} ;-79\right)$

Thus in this example, the shortest closed geodesic becomes a fiber in $\left(K_{-1} ;-79\right)$, but it is nontrivial in $S^{3}$.

However, the second shortest closed geodesic in the complement $S^{3}-K_{-1}$ is trivial in $S^{3}$ and becomes a fiber in $\left(K_{-1} ;-79\right)$, see Exercise below.

Exercise. We use a symbol $c$ to denote also the image of $c$ (the blue component) in $(c ; 1)$ and $\left(K_{-1} ;-79\right)$.

- Check that $c$ in $S^{3}-K_{-1}$ is the second shortest geodesic.

Hint: In Picture 10.5, choose Core Geodesics in View menu to get the length of the core geodesic (i.e., the core $c$ of the reglued solid torus), and compare the length in the table in the "Length spectrum" window.

Since the original $c$ is trivial in $S^{3}$, after 1-surgery along $c$, the image $c$ is still trivial in $S^{3}$. Hence the second shortest geodesic $c$ is trivial in $S^{3}$.

- Check that the second shortest geodesic $c$ becomes a fiber in $\left(K_{-1} ;-79\right)$.

Hint: Delete 1, 1 from the second row in Picture 10.3 and click Recompute button to get an information about $\left(K_{-1} ;-79\right)-c$. Then check $\pi_{1}\left(\left(K_{-1} ;-79\right)-c\right)$ by choosing Fundamental Group in View menu.

For a proper proof of this, see [22, 6.3].

Keeping the above example in mind, we propose: 
Question 3. Suppose that $K$ is a hyperbolic knot in $S^{3}$ and $(K ; r)$ is Seifert fibered. Is there a closed geodesic $c$ in $S^{3}-K$ such that:

- $c$ is unknotted in $S^{3}$, and

- $c$ is a fiber in some Seifert fibration of $(K ; r)$ ?

Question 4. Suppose that $K$ is a hyperbolic knot in $S^{3}$ and $(K ; r)$ is Seifert fibered. Then does there exist a shortest geodesic in $S^{3}-K$ which is a fiber in some Seifert fibration of $(K ; r)$ ? In particular, is a shortest geodesic in $S^{3}-K$ a trivial knot or a torus knot viewed in the Seifert fiber space $S^{3}=\left(K ; \frac{1}{0}\right)$ ?

Remark. We can check by SnapPea that shortest geodesics in $S^{3}-K$ are unknotted in $S^{3}$ for any hyperbolic knot $K$ with up to 11 crossings.

\section{SnapPea Experiment 11 (Seifert fibered surgery on (-2, 3, 7)-pretzel knot I)}

Let $K$ be the $(-2,3,7)$-pretzel knot, see Picture 11.1. It is shown in [2,p.1043] that $(K ; 17)$ is a Seifert fiber space over $S^{2}$ with three exceptional fibers of indices $2,3,5$. (In the following we assume this fact.)

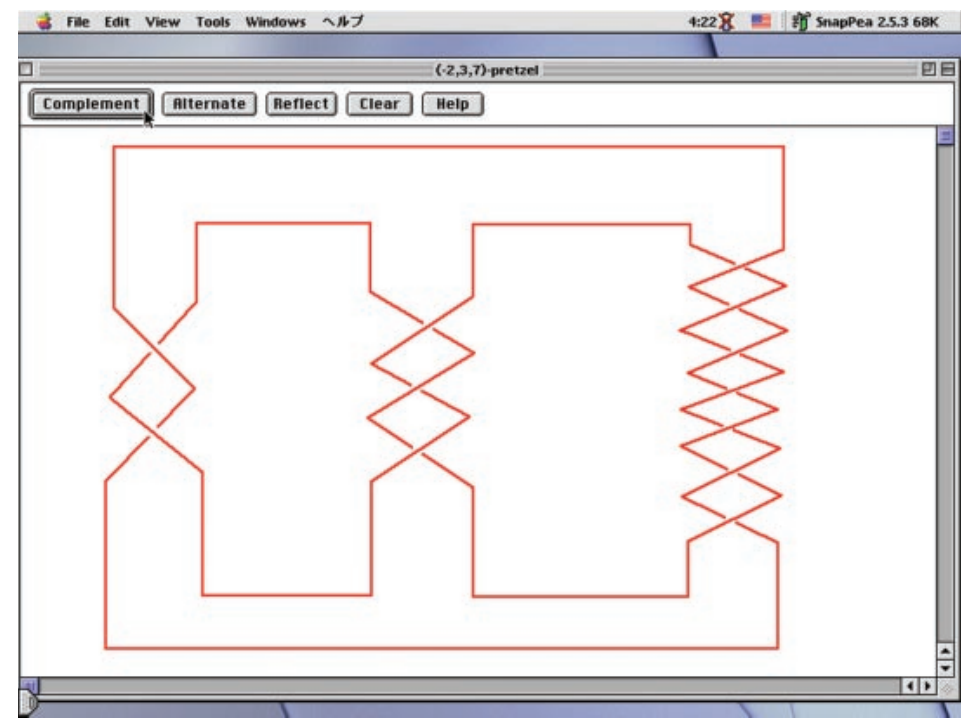

Picture 11.1.

(1) Following SnapPea Experiment 10 (3)-(5) we drill out a shortest closed geodesic and we have Pictures 11.2 and 11.3.

(2) To check whether the curve we have drilled is isotopic to the intended geodesic, perform a $\frac{1}{0}$-Dehn filling to restore the original hyperbolic structure (Picture 11.4).

(3) Apply the method in SnapPea Experiment 10 (7), (8), we have Picture 11.5. In Picture 11.5, the fundamental group of the complement of the shortest geodesic in $S^{3}$ is isomorphic to $\mathbb{Z}$. This implies by the unknotting theorem [25] that it is unknotted in $S^{3}$.

(4) Apply a similar method in SnapPea Experiment 5 (4)-(6), we have Picture 11.6 and conclude that the shortest geodesic becomes a fiber in $(K ; 17)$.

Thus Question 2 is positive for 17-surgery on $(-2,3,7)$-pretzel knot.

\section{SnapPea Experiment 12 (Seifert fibered surgery on $(-2,3,7)$-pretzel knot II)}

We continue to study 17-surgery on $(-2,3,7)$-pretzel knot $K$. Let us consider the fifth shortest geodesic $c_{5}$ in $S^{3}-K$.

(1) Following SnapPea Experiment 11 (1)-(3), we have Pictures 12.1 and 12.2. Here we select the fifth shortest closed geodesic in the "Drilling" window in Picture 12.1. (In the "Drilling" window, the fourth shortest geodesic is not offered to drill out.)

Since $\pi_{1}\left(S^{3}-c_{5}\right) \cong \mathbb{Z}$, by unknotting theorem [25], $c_{5}$ is unknotted in $S^{3}$.

(2) The structure of $(K ; 17)-c_{5}$ is given by Picture 12.3 .

In Picture 12.3 , SnapPea shows that the complement $(K ; 17)-c_{5}$ is hyperbolic, in particular, $c_{5}$ cannot become a fiber in any Seifert fibration of $(K ; 17)$. 


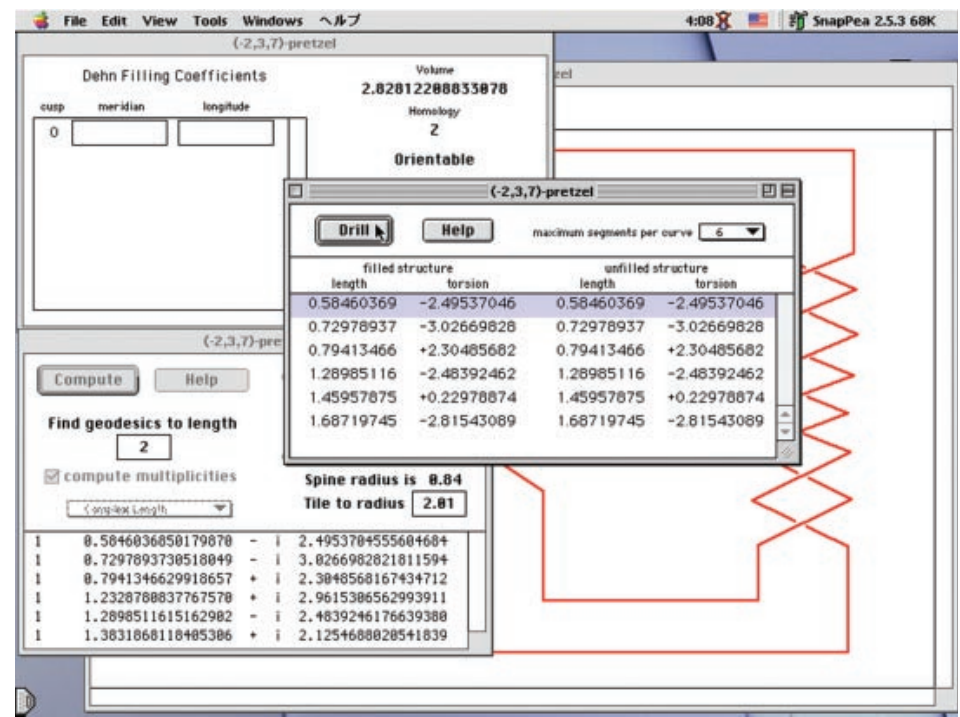

Picture 11.2.

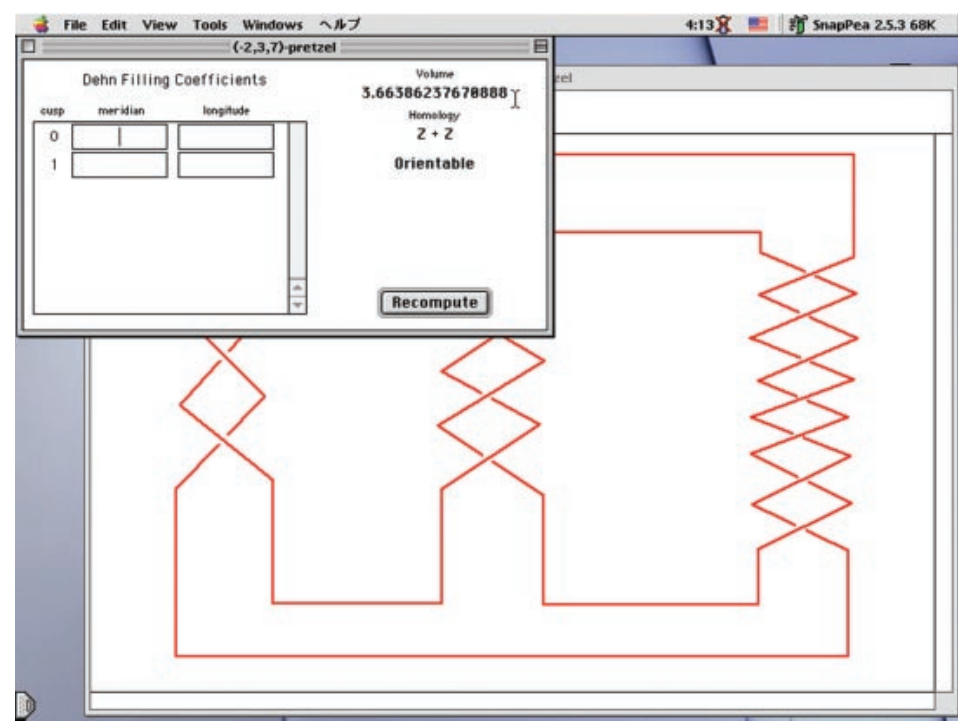

Picture 11.3.

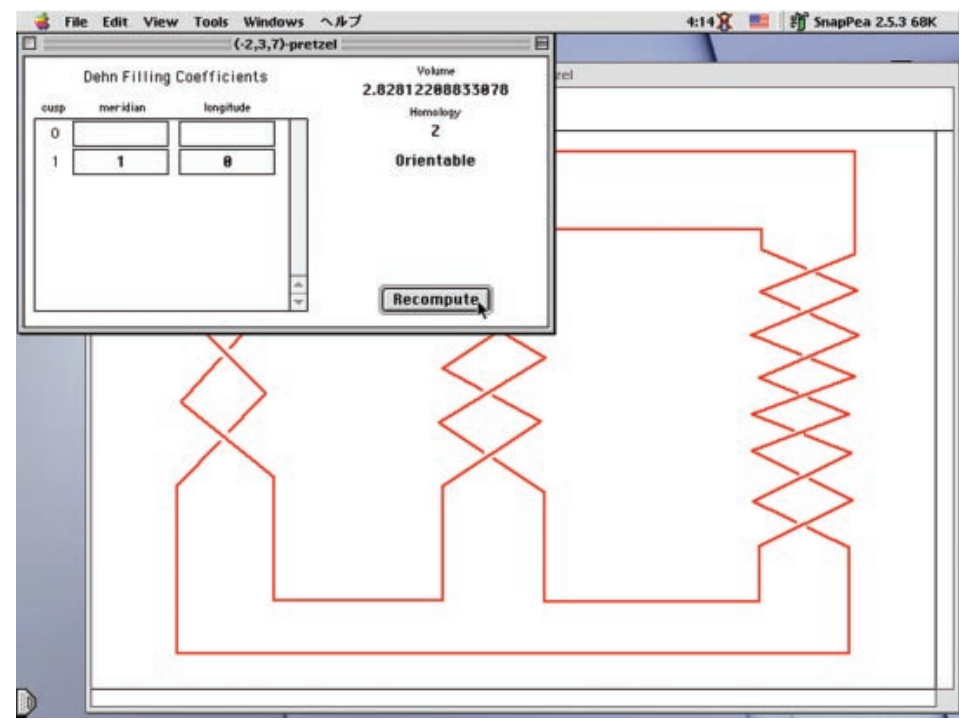

Picture 11.4 


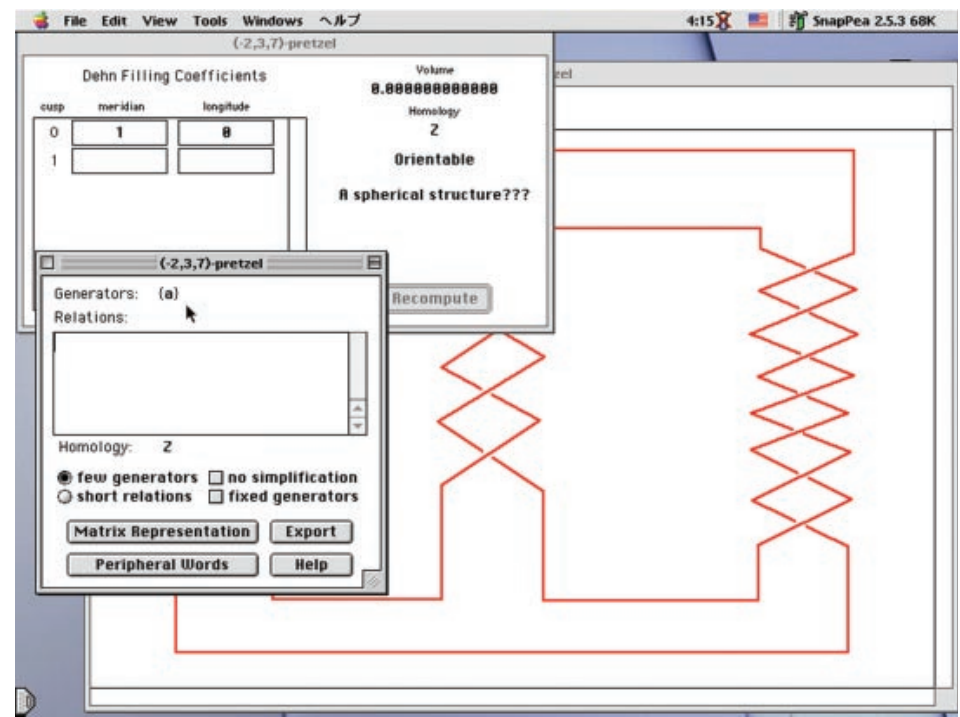

Picture 11.5.

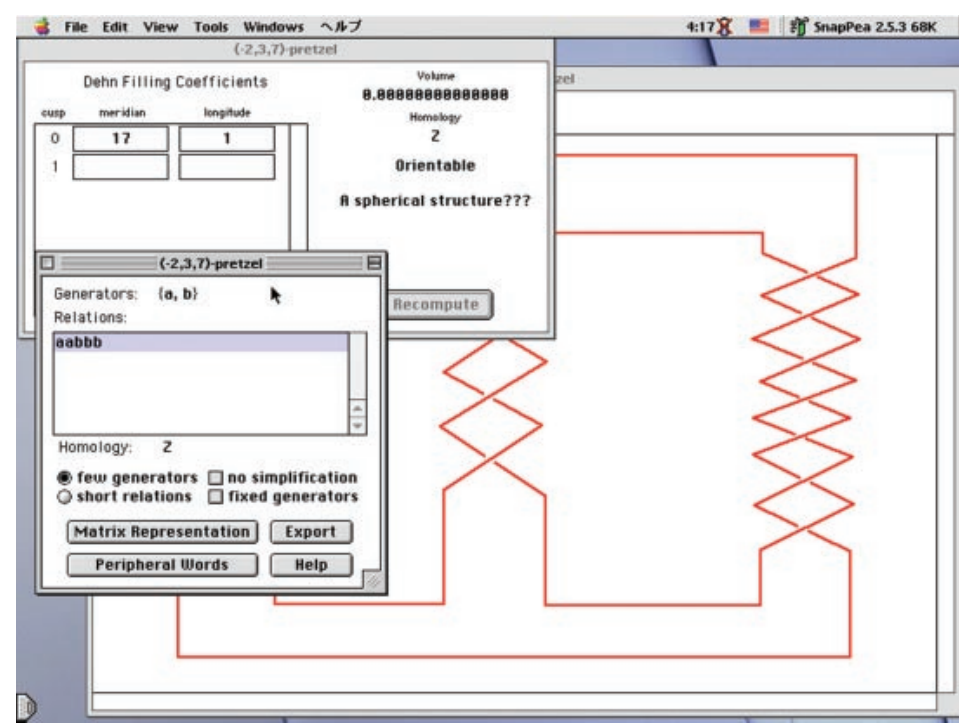

Picture 11.6.

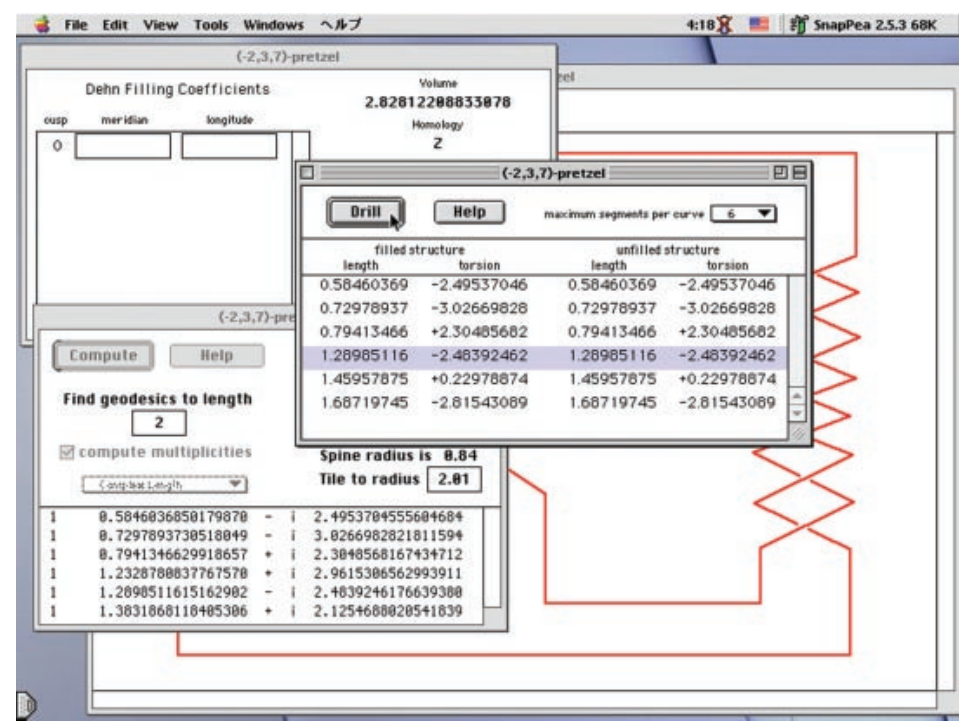

Picture 12.1. 


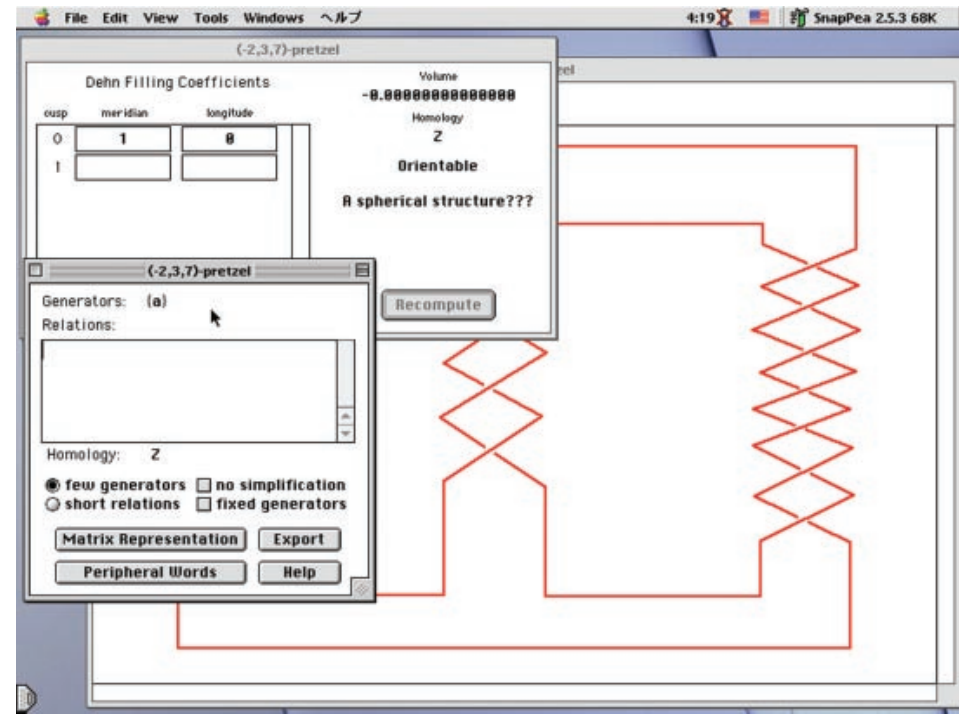

Picture 12.2.

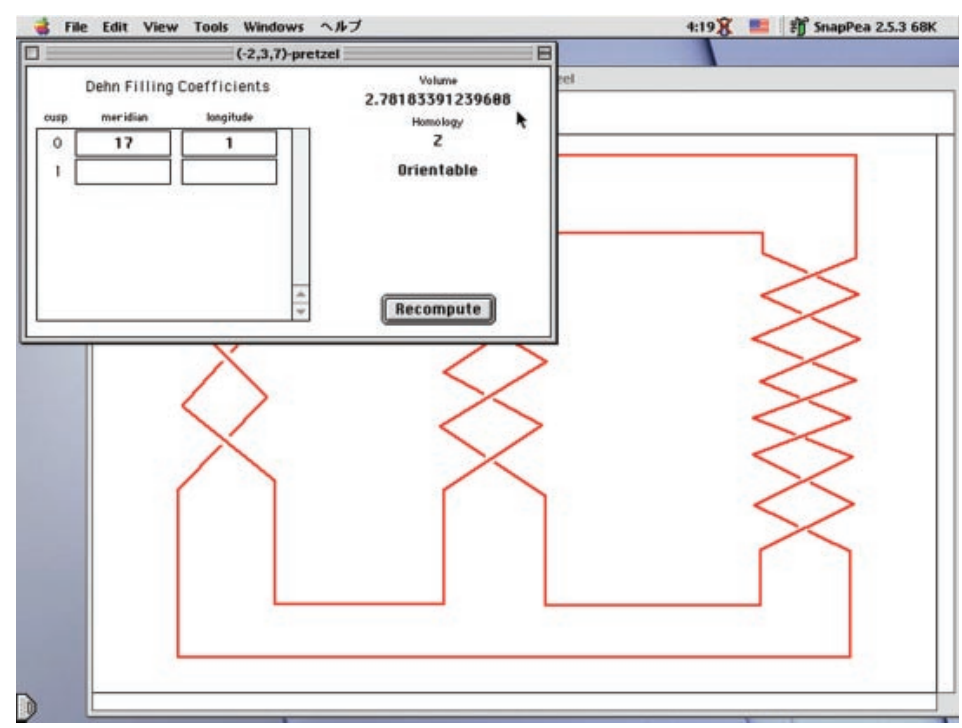

Picture 12.3.

\section{REFERENCES}

[1] Bleiler, S., and Hodgson, C., "Spherical space forms and Dehn filling," Topology, 35: 809-833 (1996).

[2] Boyer, S., and Zhang, X., "Finite surgery on knots," J. Am. Math. Soc., 9: 1005-1050 (1996).

[3] Brittenham, M., and Wu, Y. Q., “The classification of Dehn surgeries on 2-bridge knots," Comm. Anal. Geom., 9: 97-113 (2001).

[4] Casson, A., and Jungreis, D., "Convergence groups and Seifert fibered 3-manifolds," Invent. Math., 118: $441-456$ (1994).

[5] Dean, J., "Hyperbolic knots with small Seifert-fibered Dehn surgeries," Ph.D. thesis, University of Texas at Austin (1996).

[6] Eudave-Muñoz, M., "Non-hyperbolic manifolds obtained by Dehn surgery on a hyperbolic knot," in Studies in Advanced Mathematics, ed. W. Kazez, Vol. 2, Part 1, 35-61, Am. Math. Soc. and International Press (1997).

[7] Eudave-Muñoz, M., “On hyperbolic knots with Seifert fibered Dehn surgeries,” Topology Appl., 121: 119-141 (2002).

[8] Gabai, D., "Convergence groups are Fuchsian groups," Ann. Math., 136: 447-510 (1992).

[9] González-Acuña, F., and Short, H., "Knot surgery and primeness," Math. Proc. Cambridge Philos. Soc., 99: 89-102 (1986).

[10] Gordon, C. McA., "Dehn Filling; a survey," Proc. Mini Semester in Knot Theory, 129-144, Banach Center, Warsaw, Poland (1995).

[11] Gordon, C. McA., and Heil, W., "Cyclic, normal subgroups of fundamental groups of 3-manifolds," Topology, 14: 305-309 (1975).

[12] Hayashi, C., "Dehn surgery and essential annuli," Math. Proc. Cambridge Philos. Soc., 120: 127-146 (1996).

[13] Hayashi, C., and Motegi, K., "Dehn surgery on knots in solid tori creating essential annuli," Trans. Am. Math. Soc., 349: 4897-4930 (1997). 
[14] Hodgson, C. D., and Weeks, J. R., "Symmetries, isometries and length spectra of closed hyperbolic three-manifolds," Exp. Math., 3: 261-274 (1994).

[15] Jaco, W., and Shalen, P. B., "Seifert fibered spaces in 3-manifolds," Mem. Am. Math. Soc., 220, (1979).

[16] Jaco, W., "Lectures on three manifold topology," CBMS Regional Conference Series in Mathematics, Vol. 43, Am. Math. Soc. (1980).

[17] Johannson, K., "Homotopy equivalences of 3-manifolds with boundaries," Lecture Notes in Mathematics, Vol. 761, SpringerVerlag (1979).

[18] Luecke, J., "Dehn surgery on knots in the 3-sphere," Proceedings of International Congress of Mathematicians, Zürich, Switzerland, 1994, 585-594, Birkha ser Verlag (1995).

[19] Mattman, T., Miyazaki, K., and Motegi, K., "Seifert fibered surgeries which do not arise from primitive/Seifert-fibered constructions," preprint.

[20] S. Miller, M., "Geodesic knots in the figure-eight knot complement," Exp. Math., 10: 419-436 (2001).

[21] Miyazaki, K., and Motegi, K., "Seifert fibred manifolds and Dehn surgery," Topology, 36: 579-603 (1997).

[22] Miyazaki, K., and Motegi, K., "Seifert fibered manifolds and Dehn surgery III," Comm. Anal. Geom., 7: 551-582 (1999).

[23] Miyazaki, K., and Motegi, K., "On primitive/Seifert-fibered constructions," preprint.

[24] Morgan, J. and Bass, H. (eds.), The Smith conjecture, Academic Press (1984).

[25] Rolfsen, D., Knots and links, Publish or Perish, Berkeley, Calif. (1976).

[26] Scott, P., "The geometries of 3-manifolds," Bull. London Math. Soc., 15: 401-487 (1983).

[27] Thurston, W. P., The geometry and topology of 3-manifolds, Lecture notes, Princeton University (1979).

[28] Thurston, W. P., “Three dimensional manifolds, Kleinian groups and hyperbolic geometry,” Bull. Am. Math. Soc., 6: 357-381 (1982).

[29] Waldhausen, F., “Gruppen mit Zentrum und 3-dimensionale Mannigfaltigkeiten,” Topology, 6: 505-517 (1967).

[30] Weeks, J., "SnapPea": a computer program for creating and studying hyperbolic 3-manifolds, freely available from http:// humber. northnet.org/weeks/index/SnapPea.html. 